Supporting Information For:

\title{
Columnar Organization of Carbo[5]helicene Directed by Peripheral Steric Perturbation
}

Takehiro Hirao, ${ }^{\S, \dagger}$ Yudai Ono, ${ }^{\S} \uparrow$ Naomi Kawata, ${ }^{\ddagger}$ Takeharu Haino ${ }^{*, \dagger}$

†Department of Chemistry, Graduate School of Science, Hiroshima University, 1-3-1 Kagamiyama, Higashi-Hiroshima, Hiroshima, 739-8526, JAPAN

"Natural Science Center for Basic Research and Development (N-BARD), Hiroshima University, 1-3-1 Kagamiyama, Higashi-Hiroshima 739-8526, JAPAN

$\S$ These authors contributed equally. 


\section{Contents}

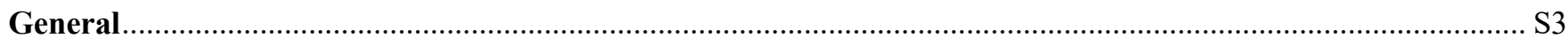

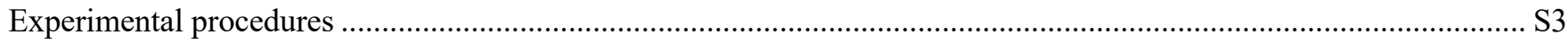

Synthetic procedure and compound data for new chemical entities...................................................................S4-S6

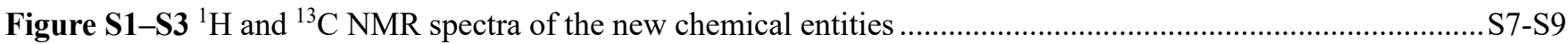

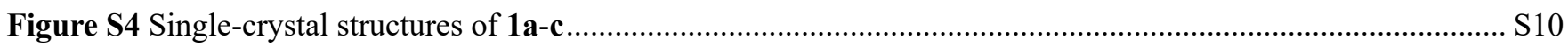

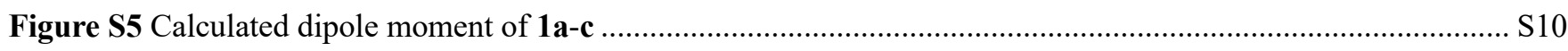

Figure S6 Determination of the free activation energy $\left(\Delta G^{\ddagger}\right)$ for the racemization .............................................. S11

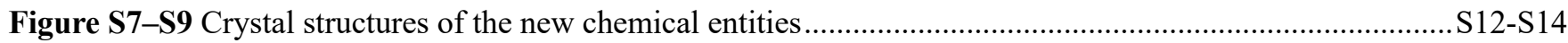

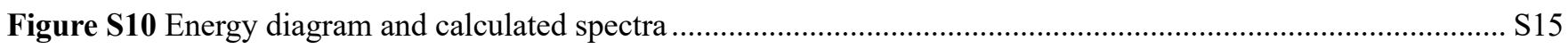

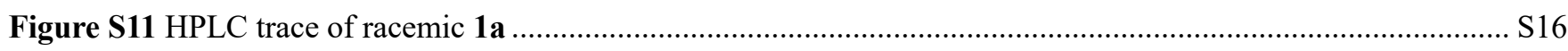

Figure S12-S14, Table S1-S3 Crystal data and structure refinement for the new chemical entities ..................S17-S22

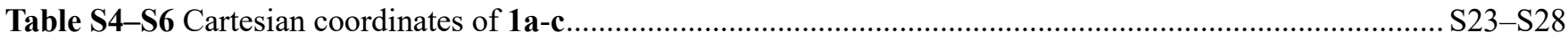

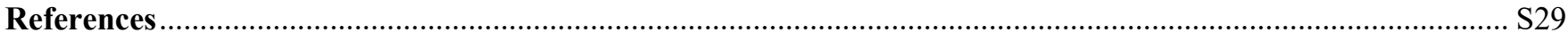


General: All reagents and solvents were commercial reagent grade and were used without further purification. ${ }^{1} \mathrm{H}$ and ${ }^{13} \mathrm{C}$ NMR spectra were recorded on a Varian mercury-300 spectrometer, and chemical shifts were reported on the delta scale in ppm relative to residual chloroform ( $\delta=7.26$ and 77.0 for ${ }^{1} \mathrm{H}$ and ${ }^{13} \mathrm{C}$, respectively). UV/vis absorption spectra were recorded on a JASCO V-560 spectrometer. Fluorescence spectra were recorded on a JASCO FP-6500 spectrometer. Chiral separations were carried out on Shimadzu LC-20AB with SPD-M20A detector and CBM-20A accessory using an analytical grade column, DAICEL CHIRALPAK IG $(4.6 \mathrm{~mm} \Phi \times 250 \mathrm{mmL}$, Particle size $5 \mu \mathrm{m})$. CD spectra was recorded on a JASCO J-1500 spectrometer. X-ray crystallographic data were collected on a Bruker SMART AEPX II ULTRA CCD diffractometer using Mo K $\alpha$ radiation $(\lambda=0.71073 \AA$ ) at $123 \mathrm{~K}$. The crystal structures were solved by SHELXT program and refined by Fourier syntheses and full-matrix least-squares procedures using the SHELXL program. The hydrogen atoms were generated geometrically. High resolution mass spectra (HRMS) were recorded on a Thermo Scientific LTQ Orbitrap XL hybrid FTMS by atmospheric pressure chemical ionization (APCI) method. Melting points (M.p.) were measured with a Yanagimoto micro melting point apparatus. 2 was synthesized according to a reported method ${ }^{1}$.

\section{Preparation of single crystals:}

$5 \mathrm{mg}$ of $\mathbf{1 a}-\mathbf{c}$ was dissolved in $1 \mathrm{~mL}$ of commercial grade chlorobenzene at room temperature. The solution was placed in a glass vial (capacity of $5 \mathrm{~mL}$ ), and then the specimen was placed on a hot plate. After being heated at $100{ }^{\circ} \mathrm{C}$ for $10 \mathrm{~min}$ without stirring under an open-air atmosphere, the hot plate was turned off to allow for reaching room temperature. The diffraction grade single crystals were grown while the temperature gradually decreased.

\section{Chiral separation on HPLC:}

$0.5 \mathrm{mg}$ of 1a was dissolved in $1 \mathrm{~mL}$ of commercial grade dichloromethane at room temperature. $15 \mu \mathrm{L}$ of the solution was injected into an HPLC system with the detector wavelength of $320 \mathrm{~nm}$ using toluene as eluent with a flow rate of $1.0 \mathrm{~mL}$ per min at room temperature. The chromatogram is shown in Figure S11.

\section{Computational details (Figure S5 and S10):}

The calculated UV/vis absorption and CD spectra of $(P)$ - and $(M)$-1a, and the total dipole moment $\left(D_{\mathrm{n}}\right)$ of 1a-c were obtained by density functional theory (DFT) at the B3LYP/6-311G(d) level using Gaussian 2009. The initial structures were generated from the crystal structure of $(P)$ - and $(M)$-1a without structure optimization. The Cartesian coordinates of the crystal structures used for the calculations and the dipole moments calculated are listed in Table S4-S6. 


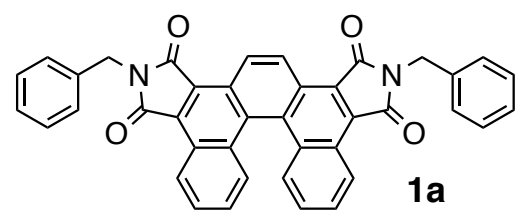

Synthesis of 2,13-bis(phenylmethyl)-dibenzo[3,4:5,6]phenanthrol[1,2-c:7,8-c']dipyrrole-1,3,12,14(2H,13H)-tetrone (1a):

To a solution of $2(50 \mathrm{mg}, 0.12 \mathrm{mmol})$ in $N$-methylpyrrolidone (NMP) $(2.0 \mathrm{~mL})$ was added $3 \mathrm{a}(39 \mathrm{mg}, 0.360 \mathrm{mmol})$ under an argon atmosphere. After being stirred for $6.5 \mathrm{~h}$ at $170{ }^{\circ} \mathrm{C}$ by oil-bath heating, the resulting mixture was diluted with dichloromethane. The organic layer was washed with $1 \mathrm{M}$ aqueous $\mathrm{HCl}$, and water, dried over anhydrous $\mathrm{Na}_{2} \mathrm{SO}_{4}$, and concentrated in vacuo. The addition of methanol to the residual NMP solution yielded the desired product as a yellow solid (45 mg, $63 \%)$.

Compound data for 1a:

M.p. $>300{ }^{\circ} \mathrm{C} ;{ }^{1} \mathrm{H}$ NMR $\left(300 \mathrm{MHz}, \mathrm{CDCl}_{3}\right): \delta 9.25$ (s, 2H), $9.15(\mathrm{~d}, 2 \mathrm{H}, J=8.3 \mathrm{~Hz}), 8.24(\mathrm{~d}, 2 \mathrm{H}, J=8.3 \mathrm{~Hz}), 7.70(\mathrm{t}, 2 \mathrm{H}, J$ $=8.3 \mathrm{~Hz}), 7.55(\mathrm{~m}, 4 \mathrm{H}), 7.28-7.42(\mathrm{~m}, 8 \mathrm{H}), 4.99(\mathrm{~s}, 4 \mathrm{H}) \mathrm{ppm} ;{ }^{13} \mathrm{C} \mathrm{NMR}\left(75 \mathrm{MHz}, \mathrm{CDCl}_{3}\right): \delta 169.2,168.9,136.5,133.9$, 130.7, 129.7, 128.9, 128.8, 128.7, 128.6, 127.9, 127.7, 127.3, 126.4, 126.3, 125.5, 125.2, 41.7 ppm; FTIR-ATR (neat): $v 3405$, 3070, 2934, 1762, 1706, 1493, 1431, 1387, 1332, 1113, 1078, 1074, 924, $837 \mathrm{~cm}^{-1}$; HRMS (APCI ${ }^{+}$) calcd. for $\mathrm{C}_{40} \mathrm{H}_{25} \mathrm{O}_{4} \mathrm{~N}_{2}$ $m / z 597.1809[\mathrm{M}+\mathrm{H}]^{+}$, found $m / z 597.1809$. 


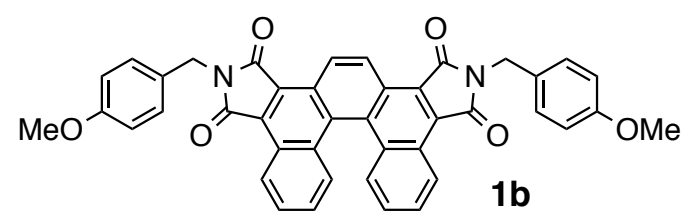

Synthesis of 2,13-bis[(4-methoxyphenyl)methyl]-dibenzo[3,4:5,6]phenanthrol[1,2-c:7,8-c']dipyrrole-1,3,12,14(2H,13H)tetrone $(\mathbf{1 b})$ :

To a solution of $2(59.8 \mathrm{mg}, 0.143 \mathrm{mmol})$ in $N$-methylpyrrolidone (NMP) $(2.0 \mathrm{~mL})$ was added $\mathbf{3 b}(68.0 \mathrm{mg}, 0.496 \mathrm{mmol})$ under an argon atmosphere. After being stirred for $6.5 \mathrm{~h}$ at $170{ }^{\circ} \mathrm{C}$ by oil-bath heating, the resulting mixture was diluted with chloroform. The organic layer was washed with $1 \mathrm{M}$ aqueous $\mathrm{HCl}$, water, dried over anhydrous $\mathrm{Na}_{2} \mathrm{SO}_{4}$, and concentrated in vасио. The addition of water to the residual NMP solution afforded the desired product as a yellow solid (88.4 mg, $94 \%)$.

\section{Compound data for $\mathbf{1 b}$ :}

M.p. $>300{ }^{\circ} \mathrm{C} ;{ }^{1} \mathrm{H}$ NMR $\left(300 \mathrm{MHz}, \mathrm{CDCl}_{3}\right): \delta 9.23$ (s, 2H), 9.14 (d, 2H, $\left.J=8.5 \mathrm{~Hz}\right), 8.23(\mathrm{~d}, 2 \mathrm{H}, J=8.5 \mathrm{~Hz}), 7.69$ (t, $2 \mathrm{H}, J$ $=8.5 \mathrm{~Hz}), 7.49(\mathrm{~d}, 4 \mathrm{H}, J=8.5 \mathrm{~Hz}), 7.37(\mathrm{~d}, 2 \mathrm{H}, J=8.5 \mathrm{~Hz}), 6.89(\mathrm{~d}, 4 \mathrm{H}, J=8.5 \mathrm{~Hz}), 4.92(\mathrm{~s}, 4 \mathrm{H}), 3.78(\mathrm{~s}, 6 \mathrm{H}) \mathrm{ppm} ;{ }^{13} \mathrm{C}$ $\operatorname{NMR}\left(75 \mathrm{MHz}, \mathrm{CDCl}_{3}\right): \delta 169.3,169.0,159.2,133.9,130.6,130.1,129.6,128.9,128.7,128.7,127.7,127.3,126.4,126.2$, 125.5, 125.2, 114.1, 55.3, 41.1 ppm; FTIR-ATR (neat): $v$ 2965, 2894, 1760, 1705, 1614, 1513, 1428, 1394, 1330, 1253, 1180, 1097, 1031, 911, $840 \mathrm{~cm}^{-1}$; HRMS (APCI ${ }^{+}$) calcd. for $\mathrm{C}_{42} \mathrm{H}_{29} \mathrm{O}_{6} \mathrm{~N}_{2} \mathrm{~m} / z 657.2020[\mathrm{M}+\mathrm{H}]^{+}$, found $\mathrm{m} / z$ 657.2023. 


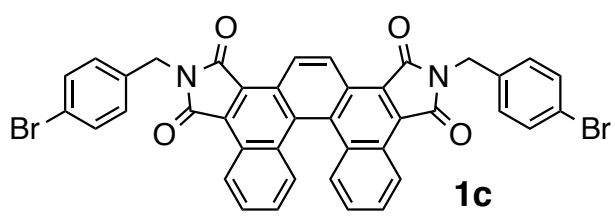

Synthesis of 2,13-bis[(4-bromophenyl)methyl]-dibenzo[3,4:5,6]phenanthrol[1,2-c:7,8-c']dipyrrole-1,3,12,14(2H,13H)tetrone (1c):

To a solution of 2 (138 mg, $0.33 \mathrm{mmol})$ in $N$-methylpyrrolidone (NMP) (4.0 mL) was added $\mathbf{3 c} \cdot$ hydrochloride $(220 \mathrm{mg}, 1.0$ mmol) under an argon atmosphere. After being stirred for $6.5 \mathrm{~h}$ at $170{ }^{\circ} \mathrm{C}$ by oil-bath heating, the resulting mixture was diluted with chloroform. The organic layer was washed with $1 \mathrm{M}$ aqueous $\mathrm{HCl}$, water, dried over anhydrous $\mathrm{Na}_{2} \mathrm{SO}_{4}$, and concentrated in vacuo. The addition of water to the residual NMP solution gave the desired product as a yellow solid (220 mg, $88 \%)$.

Compound data for $1 \mathbf{c}$ :

M.p. $>300{ }^{\circ} \mathrm{C}$; ${ }^{1} \mathrm{H}$ NMR $\left(300 \mathrm{MHz}, \mathrm{CDCl}_{3}\right): \delta 9.23$ (s, 2H), 9.13 (d, 2H, $\left.J=8.4 \mathrm{~Hz}\right), 8.24(\mathrm{~d}, 2 \mathrm{H}, J=8.4 \mathrm{~Hz}), 7.70(\mathrm{t}, 2 \mathrm{H}, J$ $=8.4 \mathrm{~Hz}), 7.49(\mathrm{~d}, 4 \mathrm{H}, J=8.4 \mathrm{~Hz}), 7.34-7.44(\mathrm{~m}, 6 \mathrm{H}), 4.93(\mathrm{~s}, 4 \mathrm{H}) \mathrm{ppm} ;{ }^{13} \mathrm{C} \mathrm{NMR}\left(75 \mathrm{MHz}, \mathrm{CDCl}_{3}\right): \delta 169.1,168.8,135.4$, $133.9,131.9,130.8,130.4,129.7,129.0,128.7,127.8,127.3,126.3,126.2,125.5,125.2,122.0,41.1$ ppm; FTIR-ATR (neat): $v$ 3088, 2970, 1764, 1708, 1486, 1435, 1385, 1334, 1264, 1183, 1093, 1063, 1013, 918, $839 \mathrm{~cm}^{-1}$; HRMS (APCI ${ }^{+}$) calcd. for $\mathrm{C}_{40} \mathrm{H}_{23} \mathrm{O}_{4} \mathrm{~N}_{2} \mathrm{Br}_{2} m / z 753.0012[\mathrm{M}+\mathrm{H}]^{+}$, found $m / z 753.0025$. 

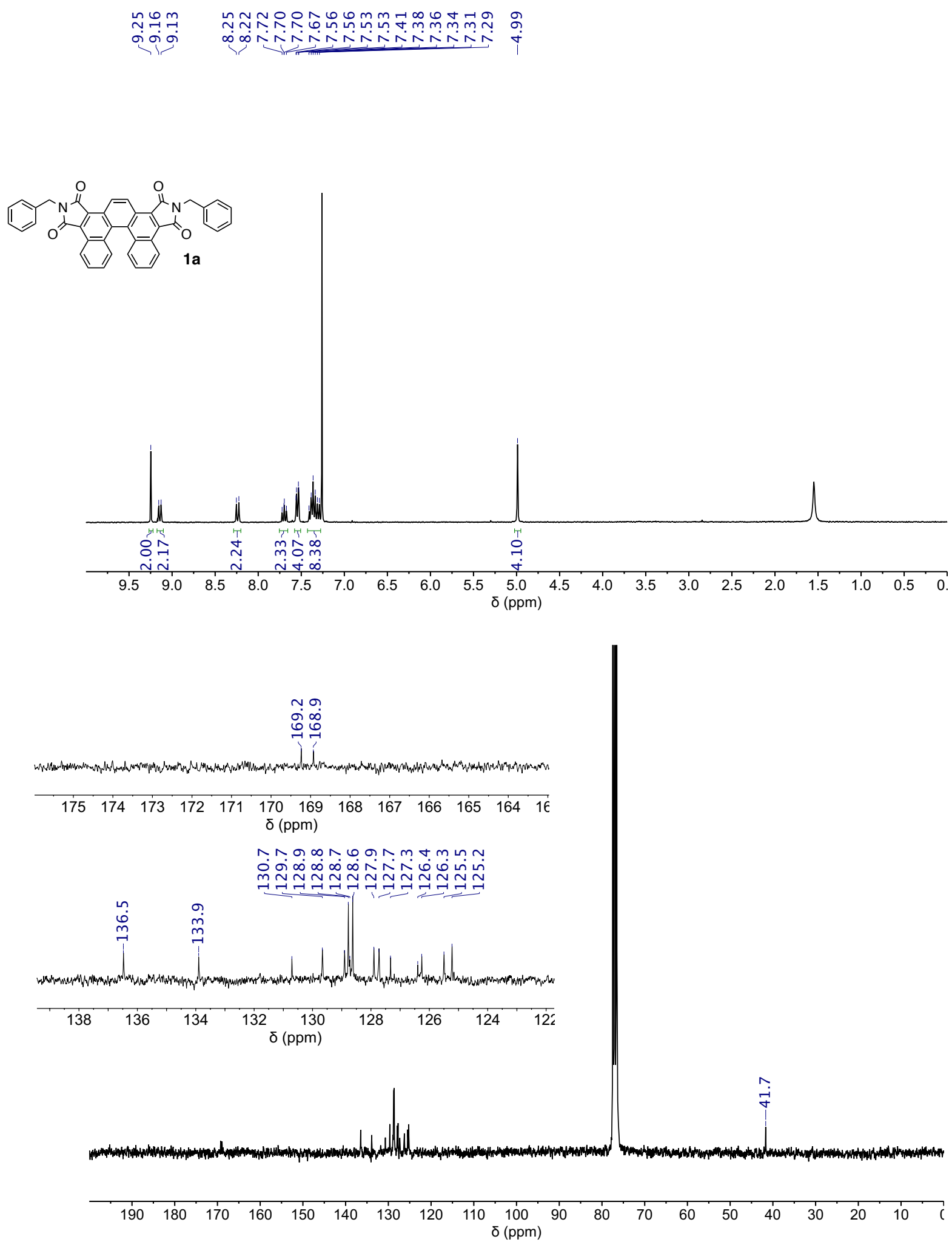

Figure S1. ${ }^{1} \mathrm{H}$ and ${ }^{13} \mathrm{C}$ NMR spectra of $1 \mathrm{a}\left(300 \mathrm{MHz}\right.$ for ${ }^{1} \mathrm{H}, 75 \mathrm{MHz}$ for ${ }^{13} \mathrm{C}$, chloroform- $d$ ). 

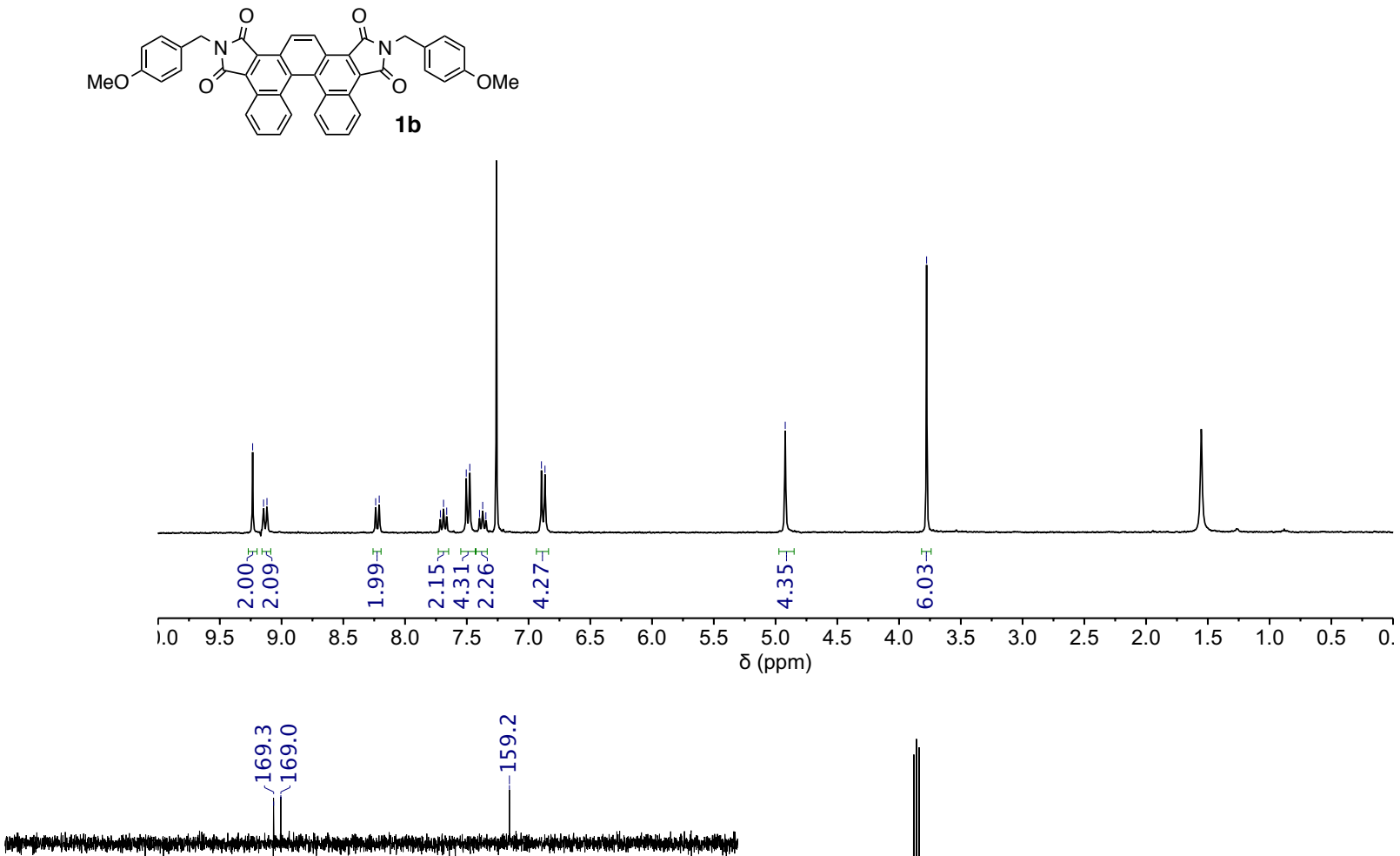

$\begin{array}{llllllllllllllll}180 & 178 & 176 & 174 & 172 & 170 & 168 & 166 & 164 & 162 & 160 & 158 & 156 & 154 & 152 & 15\end{array}$ $\delta(\mathrm{ppm})$
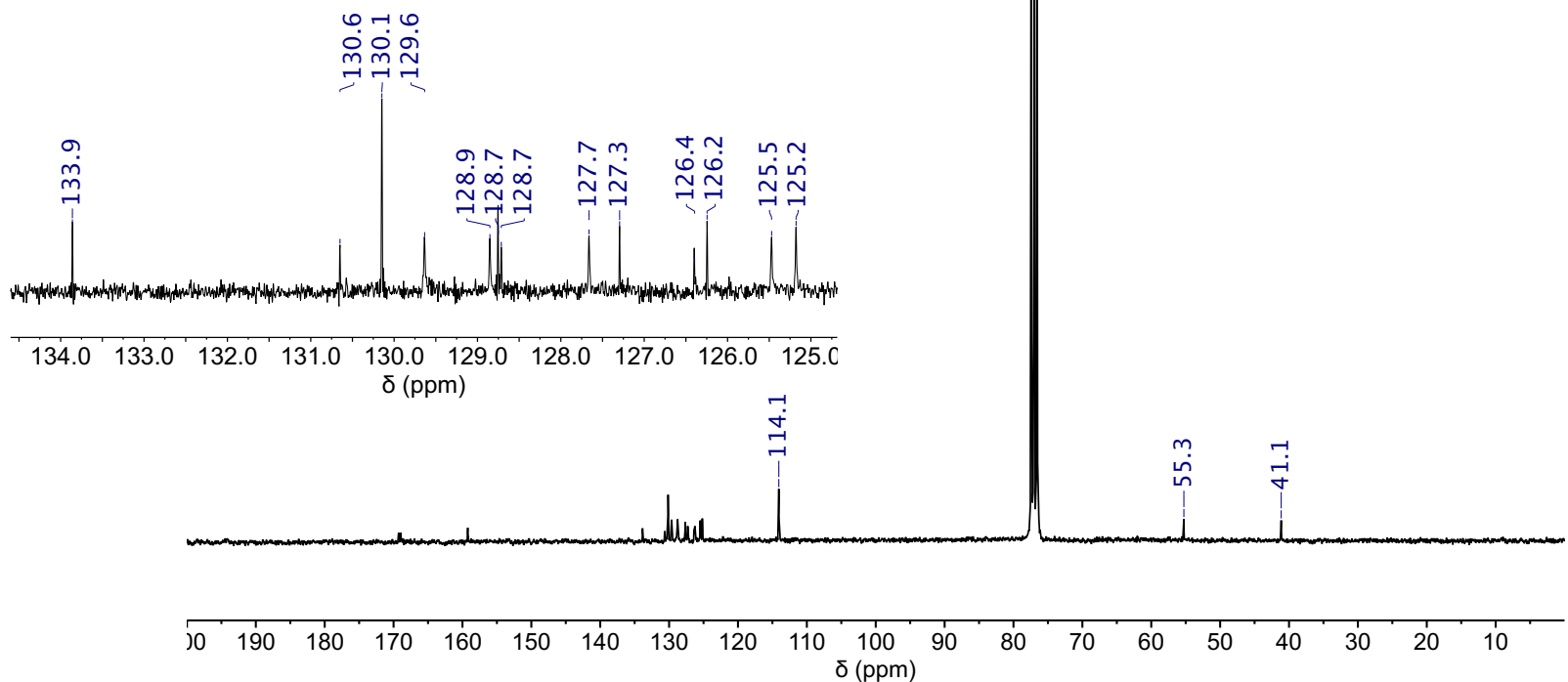

Figure S2. ${ }^{1} \mathrm{H}$ and ${ }^{13} \mathrm{C}$ NMR spectra of $\mathbf{1 b}\left(300 \mathrm{MHz}\right.$ for ${ }^{1} \mathrm{H}, 75 \mathrm{MHz}$ for ${ }^{13} \mathrm{C}$, chloroform- $d$ ). 

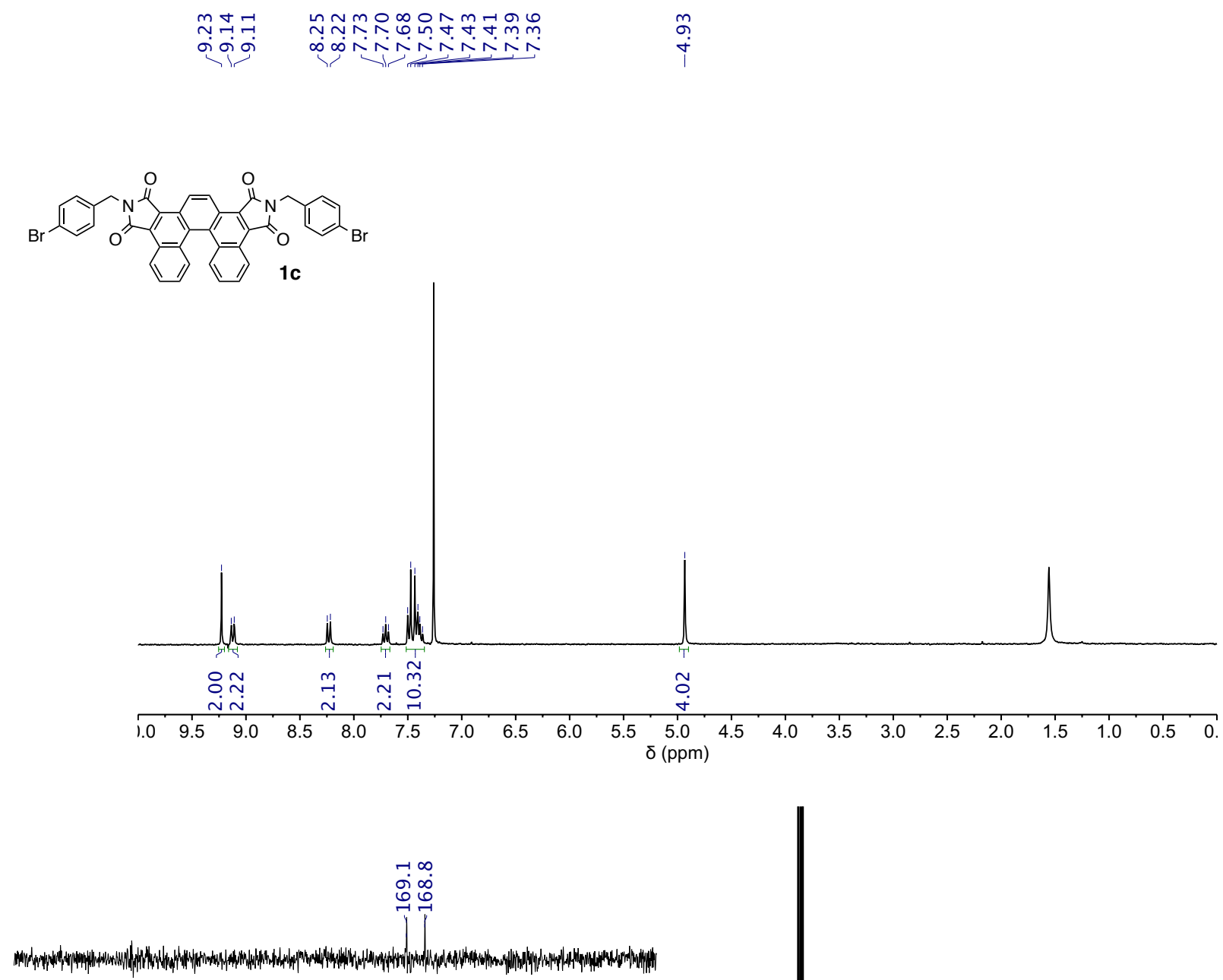

$\begin{array}{llllllllllll}175.0 & 174.0 & 173.0 & 172.0 & 171.0 & 170.0 & 169.0 & 168.0 & 167.0 & 166.0 & 165\end{array}$ $\delta(\mathrm{ppm})$

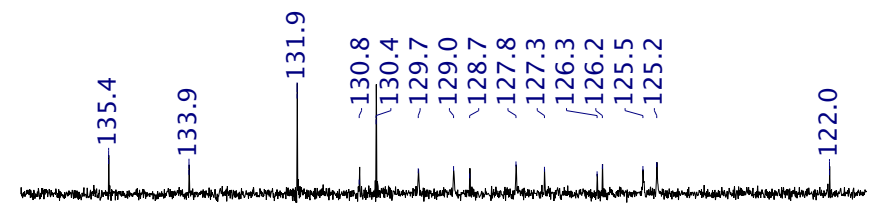

$\begin{array}{lllllllllllllllll}37 & 136 & 135 & 134 & 133 & 132 & 131 & 130 & 129 & 128 & 127 & 126 & 125 & 124 & 123 & 122 \\ \delta(\mathrm{ppm}) & & & & \end{array}$
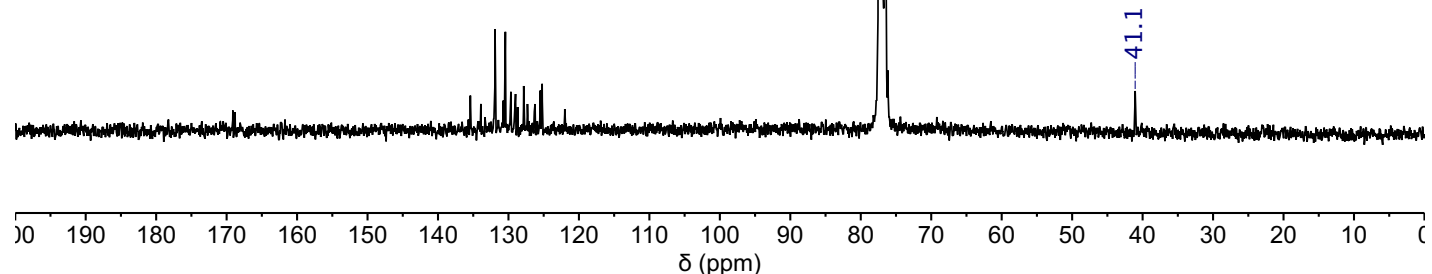

Figure S3. ${ }^{1} \mathrm{H}$ and ${ }^{13} \mathrm{C}$ NMR spectra of $1 \mathrm{c}\left(300 \mathrm{MHz}\right.$ for ${ }^{1} \mathrm{H}, 75 \mathrm{MHz}$ for ${ }^{13} \mathrm{C}$, chloroform- $d$ ). 
(a)

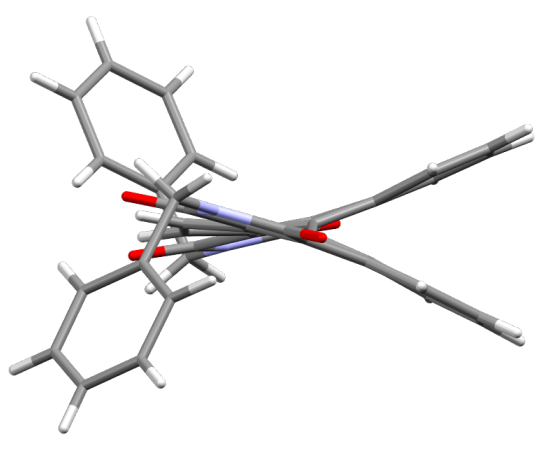

(b)

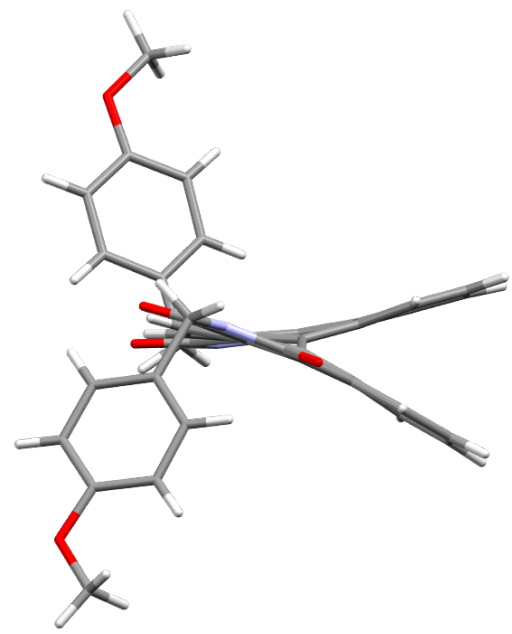

(c)

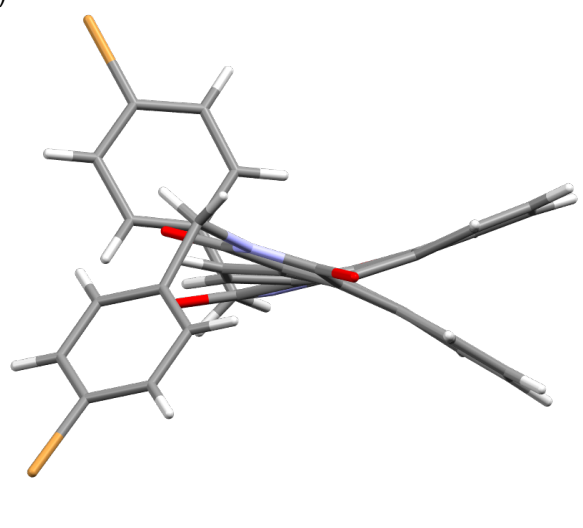

Figure S4. Side views of the structures of (a) $(M)-1 \mathbf{a},(\mathrm{b})(M)-\mathbf{1 b}$, and (c) (M)-1c obtained by single-crystal X-ray diffraction.

(a)

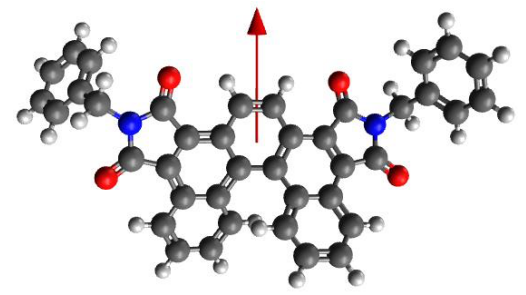

(c)

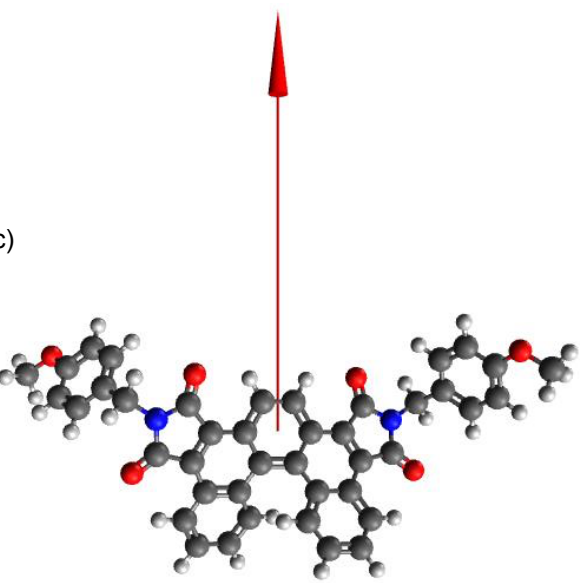

(e)

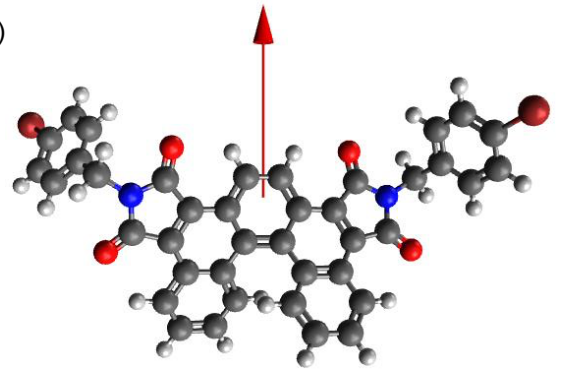

(b)
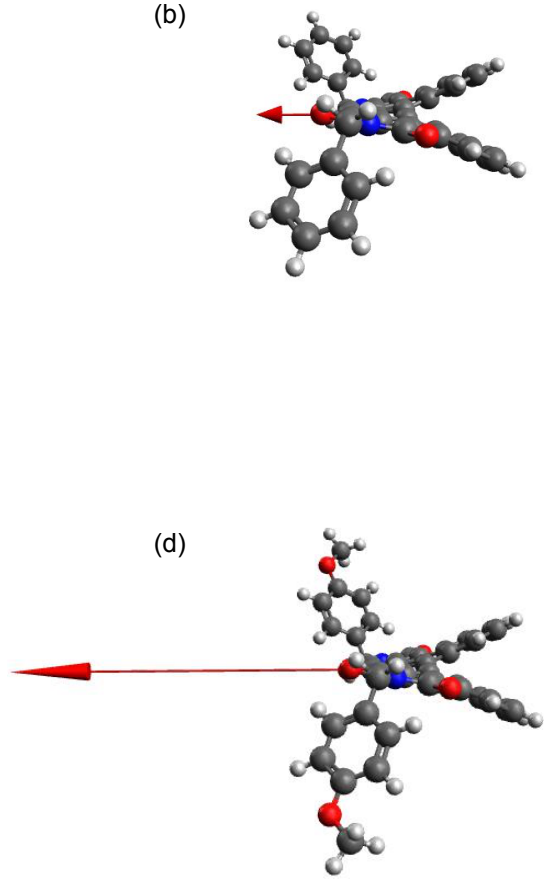

(f)

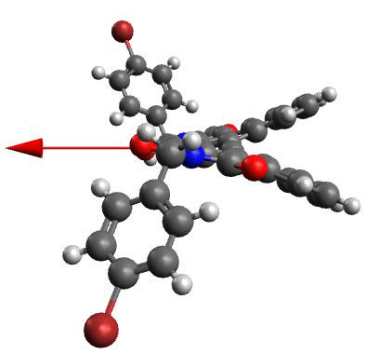

Figure S5. Calculated dipole moment of (a) 1a, (b) 1b, and (c) 1c indicated by red arrows. 


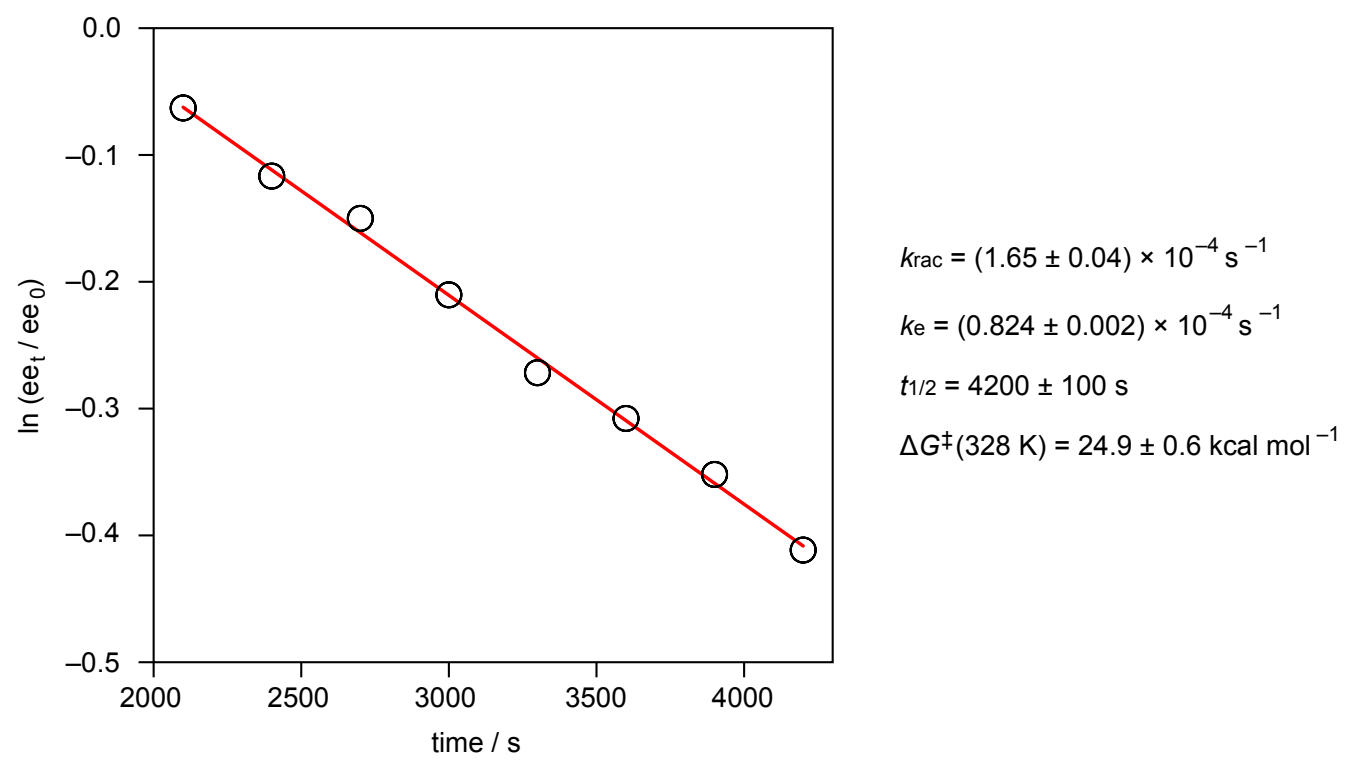

Figure S6. Plot of $\ln \left(e_{t} / e_{0}\right)$ against time, where $e_{t}$ and $e_{0}$ denote $C D$ intensity of $(M)-1 \mathbf{a}\left(0.60 \times 10^{-5} \mathrm{~mol} \mathrm{~L}^{-1}\right.$, in chloroform at $328 \mathrm{~K})$ observed at each time and CD intensity of $(M)-1 \mathrm{a}\left(0.60 \times 10^{-5} \mathrm{~mol} \mathrm{~L}^{-1}\right.$, in chloroform at $\left.293 \mathrm{~K}\right)$, respectively. The time dependent $\mathrm{CD}$ intensity changes of $(M)$-1a were monitored every $300 \mathrm{~s}$ at a single wavelength of 255 nm.

(M)-1a, obtained by chiral HPLC, was used for the determination of the Gibbs activation energy of enantiomerization $\Delta G^{\ddagger}(T){ }^{2}$ According to a relation of $\ln \left(\mathrm{ee}_{\mathrm{t}} / \mathrm{ee}_{0}\right)=-k_{\mathrm{rac}}$, where $k_{\text {rac }}$ and $t$ indicate a rate constant of racemization and time, respectively, the slope given by the plot (Fig. S6) indicates $k_{\text {rac }}$. A half-time of the process $\left(t_{1 / 2}\right)$ can be estimated by a relation, $t_{1 / 2}=\ln \left(2 / k_{\text {rac }}\right)$. Eyring equation guarantees a relation between $\Delta G^{\ddagger}(T)$ and $k_{\mathrm{e}}$ to be $\Delta G^{\ddagger}(T)=-\mathrm{R} T \ln \left(k_{\mathrm{e}} \mathrm{h} / \mathrm{K}_{\mathrm{B}} T\right)$, where $k_{\mathrm{e}}$ is a rate constant of enantiomerizatioin $\left(k_{\mathrm{e}}=k_{\mathrm{rad}} / 2\right), \mathrm{R}$ is a gas constant, $T$ is absolute temperature, $\mathrm{h}$ is a Planck constant, $\mathrm{k}_{\mathrm{B}}$ is a Boltzmann constant, and $\mathrm{K}$ is a transmission coefficient. Here, $\mathrm{K}_{\mathrm{K}}=0.5$ is used for the calculation since the enantiomerization process is a reversible first order reaction. 
(a)

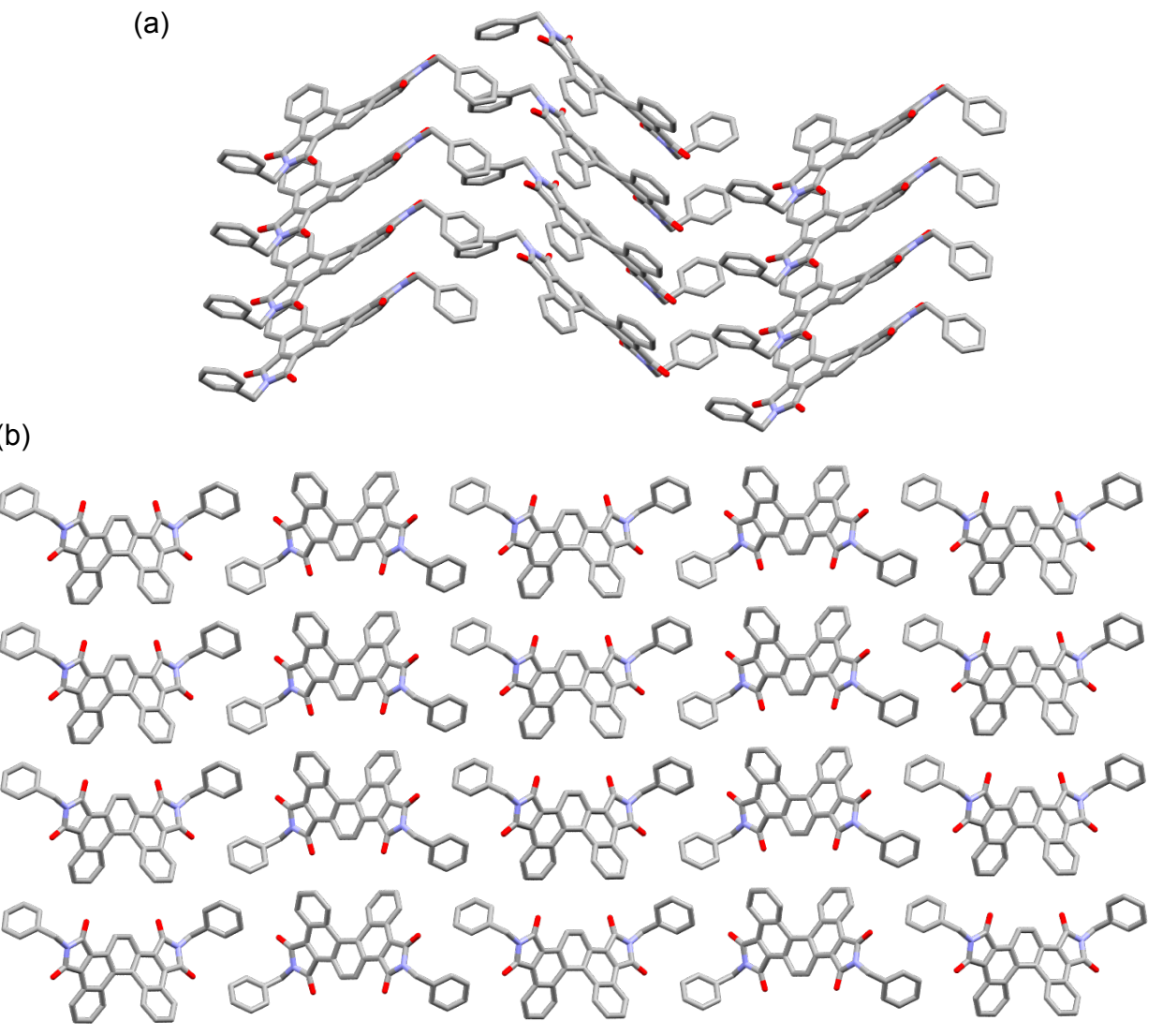

(b) (c)

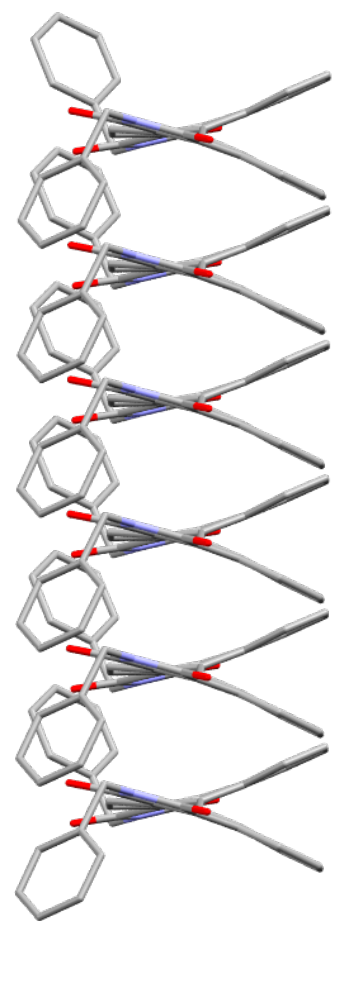

Figure S7. (a) Front view, (b) top view, and (c) side view of crystal structure of 1a. All hydrogen atoms are omitted for clarity 
(a)

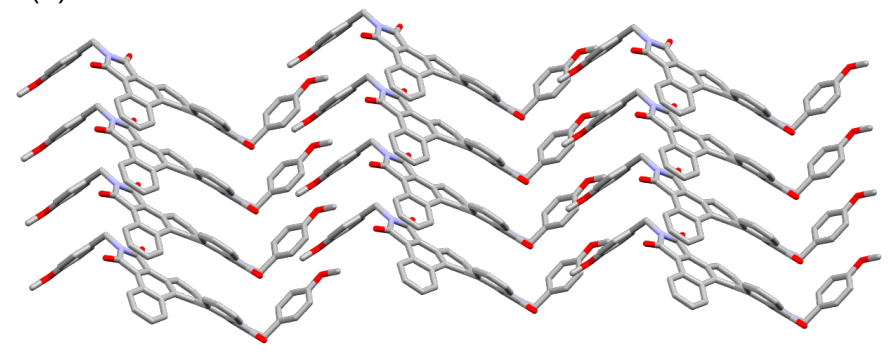

(b)
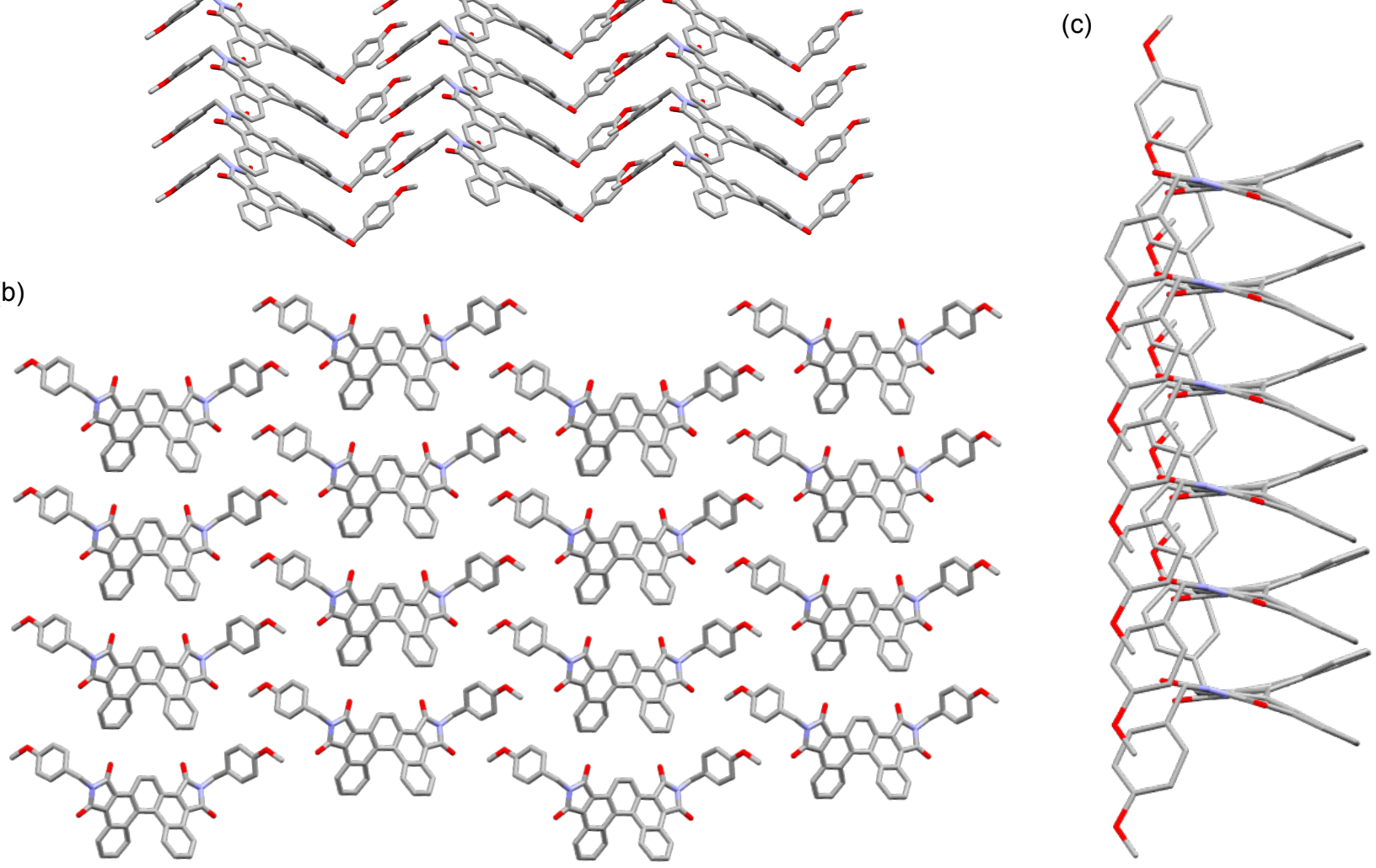

Figure S8. (a) Front view, (b) top view, and (c) side view of crystal structure of $\mathbf{1 b}$. All hydrogen atoms are omitted for clarity 
(a)

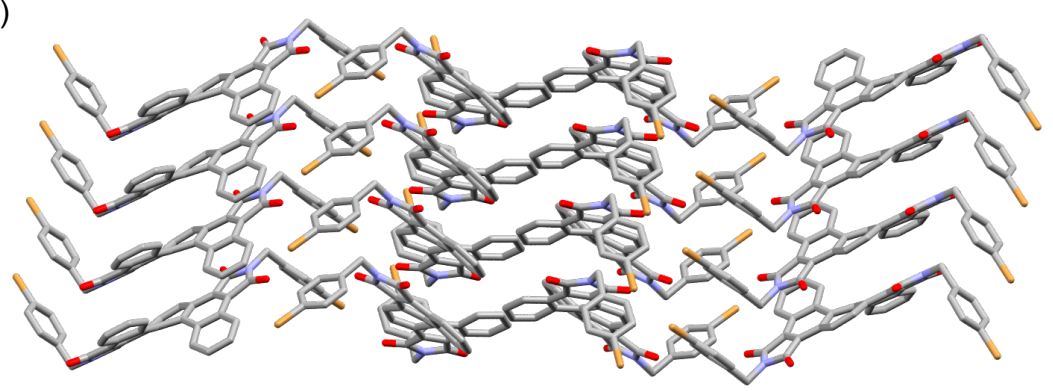

(b)

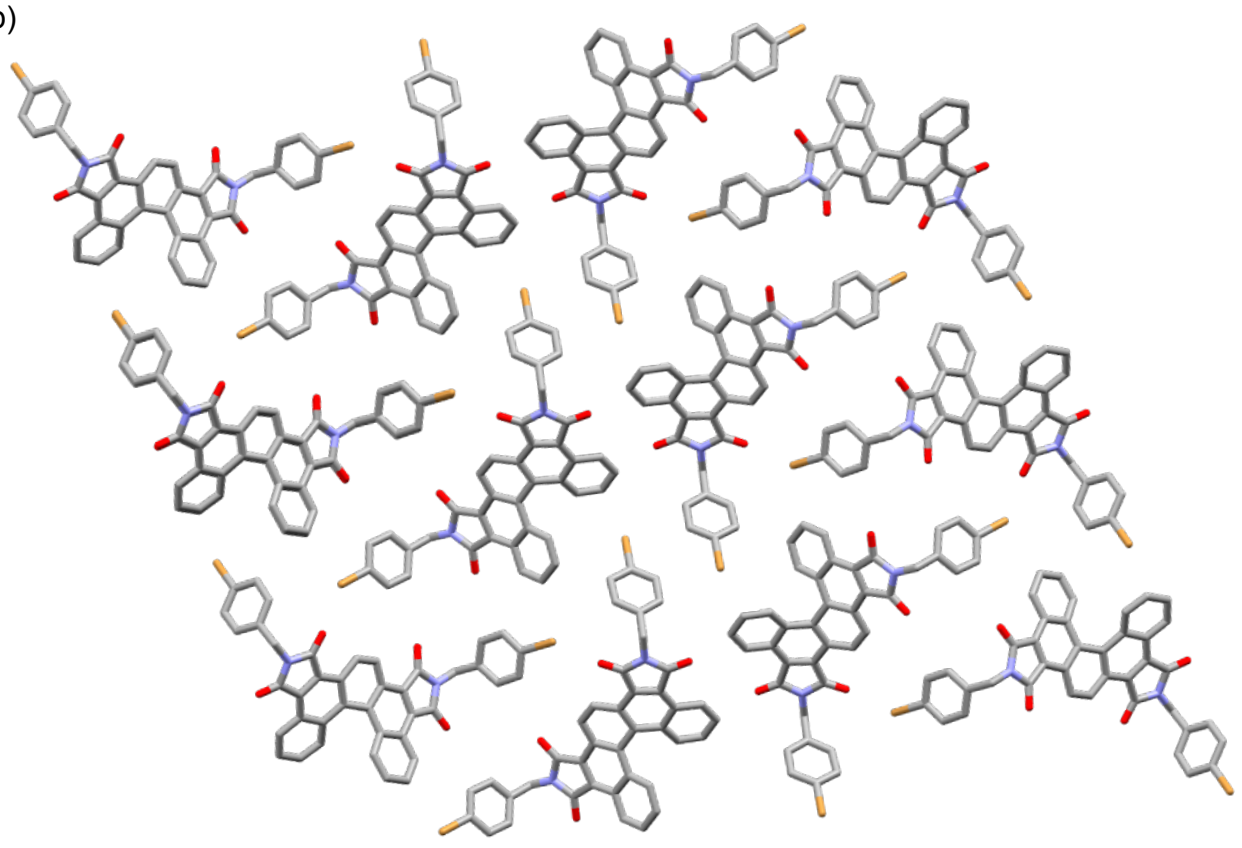

(c)

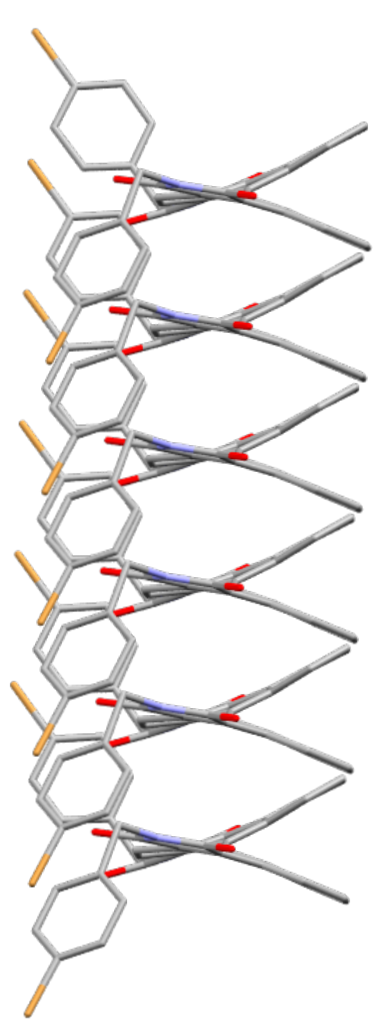

Figure S9. (a) Front view, (b) top view, and (c) side view of crystal structure of 1c. All hydrogen atoms are omitted for clarity. 

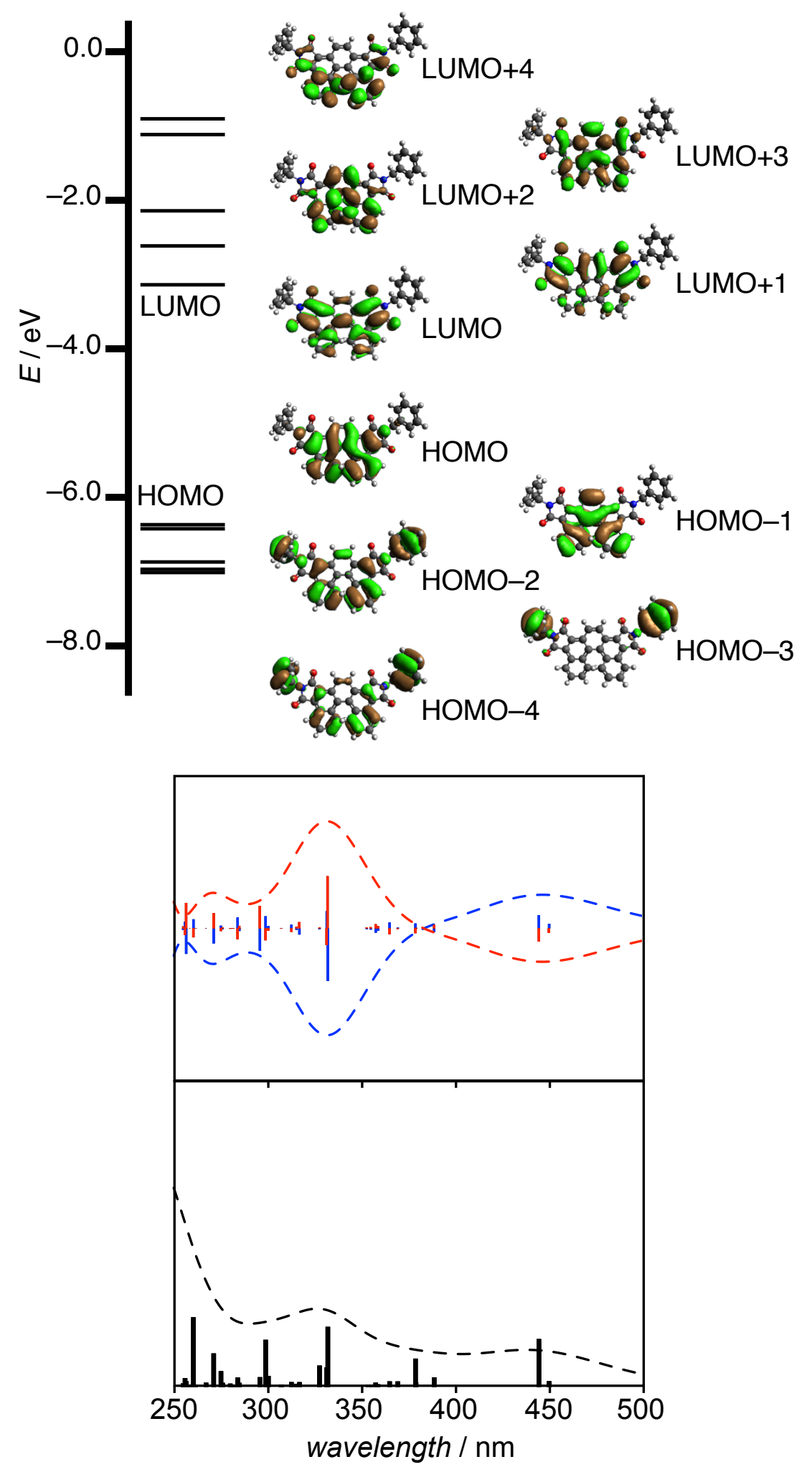

Figure S10. (Top) Energy diagram of 1a by TD-DFT at B3LYP/6-311(d) level. (Bottom) Calculated UV/vis absorption and CD spectra of 1a. The broken red and blue lines indicate calculated CD spectra of $(P)$-1a and $(M)$-1a, respectively. 
(a)

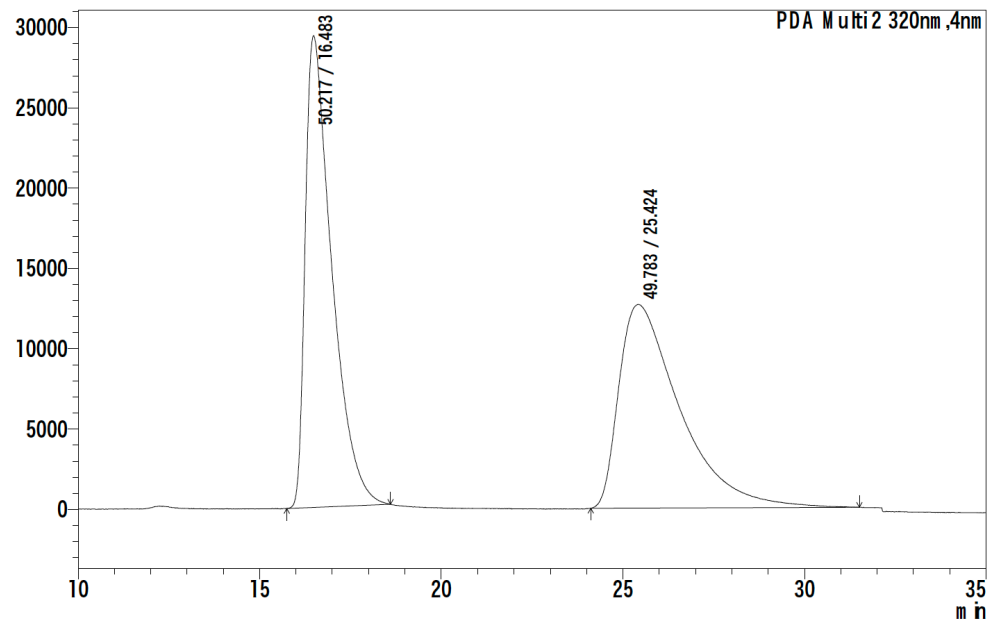

(b)

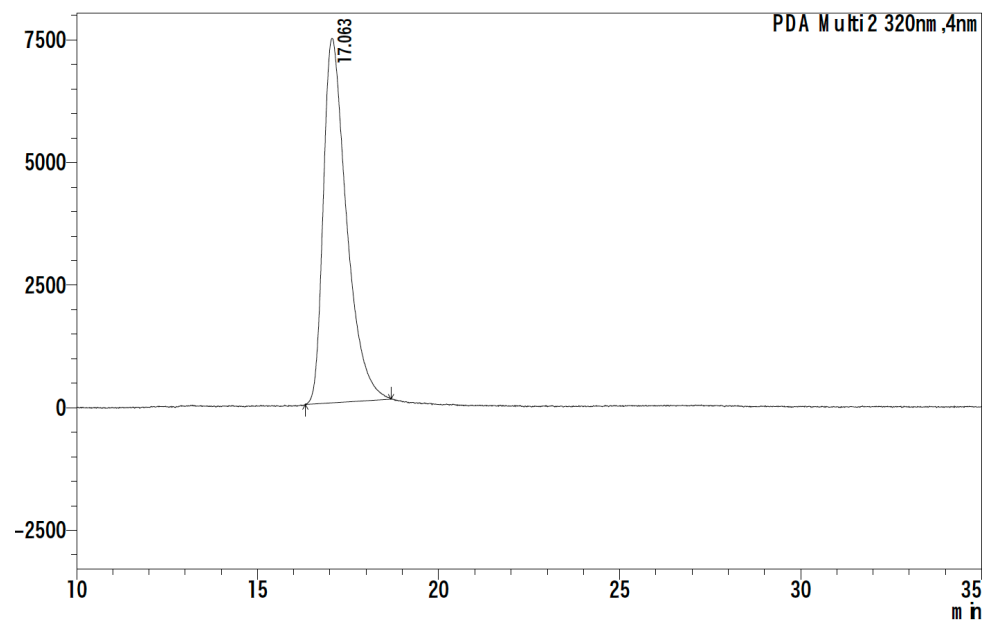

(c)

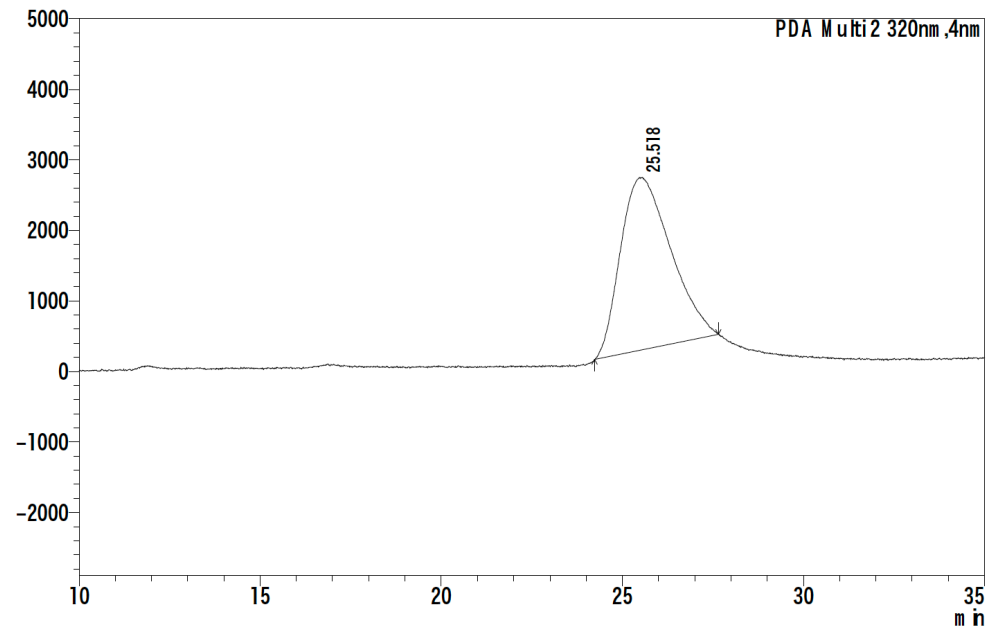

Figure S11. HPLC trace of (a) racemic 1a, (b) separated $(P)$-1a and (c) separated (M)-1a: eluent, toluene; the flow rate, 1.0 $\mathrm{mL} \mathrm{min}^{-1}$; the detector wavelength, $320 \mathrm{~nm}$. The former and the latter peaks correspond to $(P)-\mathbf{1 a}$ and $(M)-\mathbf{1 a}$, respectively. 


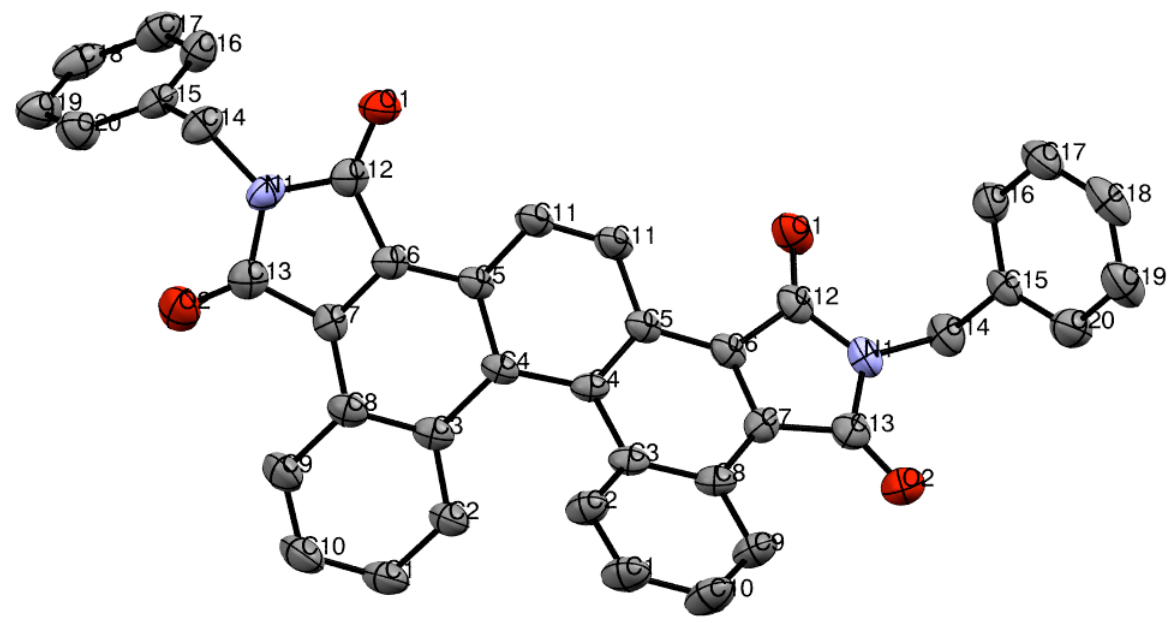

Figure S12. View of 1a showing the atom labeling scheme. Displacement ellipsoids are scaled to the $50 \%$ probability level. 
Table S1. Crystal data and structure refinement for 1a.

Chemical formula C40 H24 N2 O4

Formula weight 596.61

Temperature $123 \mathrm{~K}$

Wavelength $0.71073 \AA$

Crystal system orthorhombic

Space group $P 2{ }_{1} 2{ }_{1}$

Unit cell dimensions $a=31.545(19) \AA$

$$
\begin{aligned}
& b=4.600(3) \AA \\
& c=9.755(6) \AA
\end{aligned}
$$

Volume 1415.5(15) $\AA^{3}$

Z2

Density (calculated) $1.400 \mathrm{~g} \mathrm{~cm}^{-3}$

Absorption coefficient $0.091 \mathrm{~mm}^{-1}$

F (000) 620

Crystal size $0.20 \times 0.03 \times 0.01 \mathrm{~mm}$

Theta range for data collection 2.09 to $25.04^{\circ}$

Index ranges $-37<=h<=37,-5<=k<=5,-11<=k<=11$

Reflections collected 13622

Independent reflections 2505

$R$ (int) $=0.1021$

Completeness to theta $=25.04^{\circ} 99.9 \%$

Absorption correction multi-scan

Max. and min. transmission 1.00 and 0.84

Refinement method SHELXL

Data / restraints / parameters 2505 / 0 / 208

Goodness-of-fit on $F 21.013$

$\mathrm{R}$ indices $[\mathrm{I}>2 \operatorname{sigma}(\mathrm{I})] R 1=0.0526 w R 2=0.1069$

$\mathrm{R}$ indices (all data) $R 1=0.0805 w R 2=0.0952$

Absolute structure parameter 1.4(10)

Largest diff. peak and hole 0.166 and -0.184 e $\AA^{-3}$

CCDC \#1991476 


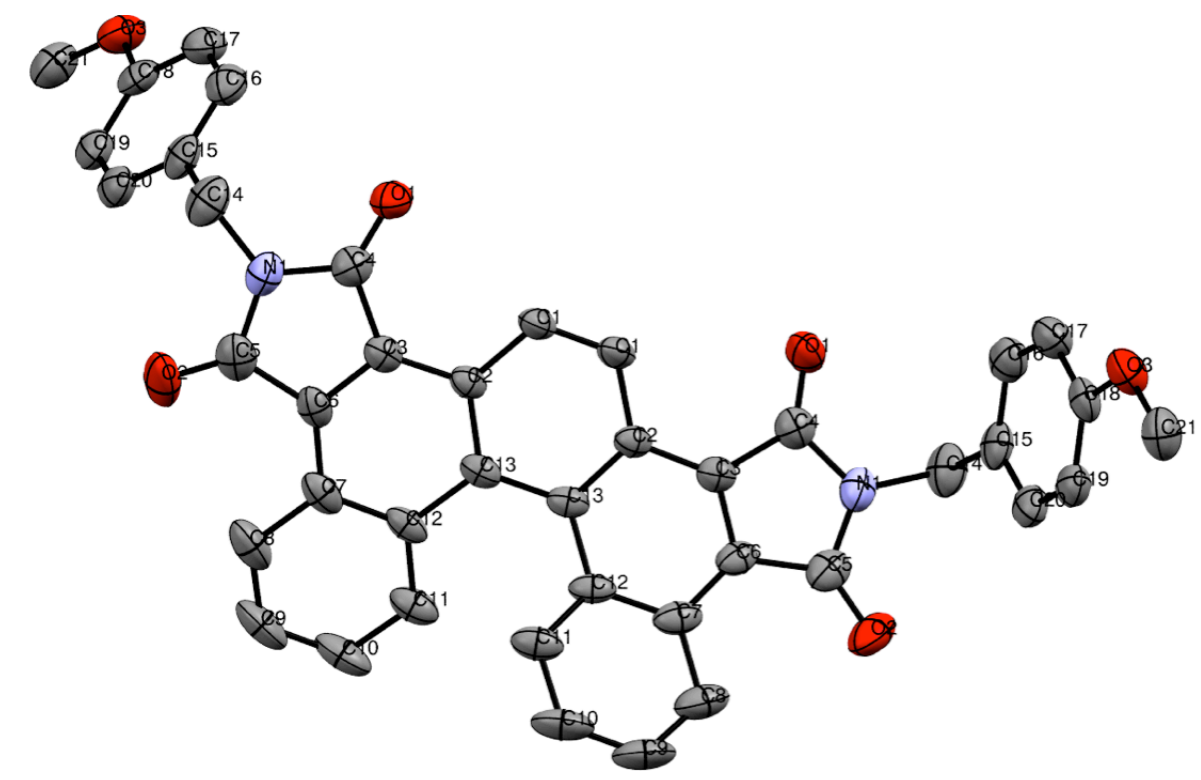

Figure S13. View of $\mathbf{1 b}$ showing the atom labeling scheme. Displacement ellipsoids are scaled to the $50 \%$ probability level. 
Table S2. Crystal data and structure refinement for $\mathbf{1 b}$.

Chemical formula C42 H28 N2 O6

Formula weight 656.68

Temperature $123 \mathrm{~K}$

Wavelength $0.71073 \AA$

Crystal system Monoclinic

Space group $C 2$

Unit cell dimensions $a=35.678(10) \AA$

$$
\begin{aligned}
& b=9.681(3) \AA \\
& c=4.4524(12) \AA \\
& \beta=91.907(4)^{\circ}
\end{aligned}
$$

Volume 1537.0(7) $\AA^{3}$

$Z 2$

Density (calculated) $1.419 \mathrm{~g} \mathrm{~cm}^{-3}$

Absorption coefficient $0.096 \mathrm{~mm}^{-1}$

F (000) 684

Crystal size $0.30 \times 0.20 \times 0.05 \mathrm{~mm}$

Theta range for data collection 2.18 to $27.94^{\circ}$

Index ranges $-46<=h<=46,-9<=k<=12,-5<=l<=5$

Reflections collected 4577

Independent reflections 2668

$R$ (int) $=0.0265$

Completeness to theta $=27.94^{\circ} 98.9 \%$

Absorption correction multi-scan

Max. and min. transmission 0.99 and 0.84

Refinement method SHELXL

Data / restraints / parameters 2668 / 1 / 227

Goodness-of-fit on $F 21.030$

$\mathrm{R}$ indices [I $>2 \operatorname{sigma}(\mathrm{I})] R 1=0.0426 w R 2=0.0816$

$\mathrm{R}$ indices (all data) $R 1=0.0682 w R 2=0.0914$

Absolute structure parameter $-0.5(10)$

Largest diff. peak and hole 0.194 and -0.182 e $\AA^{-3}$

CCDC \#1991475 


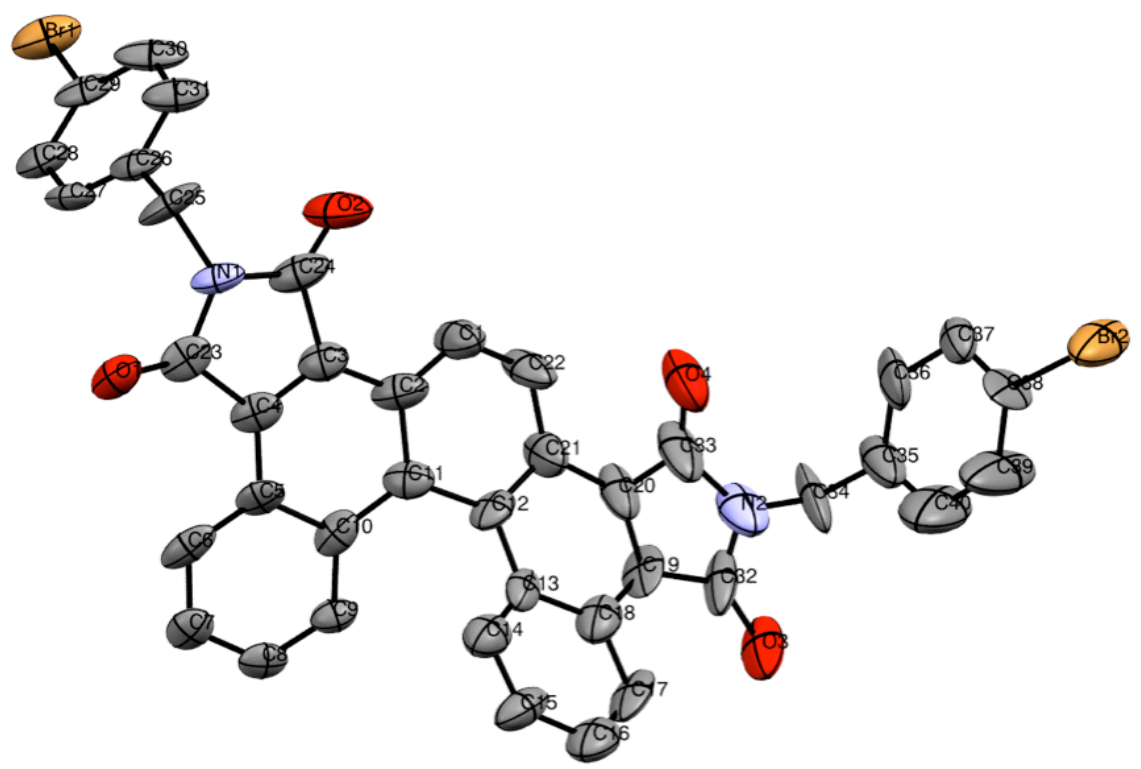

Figure S14. View of 1c showing the atom labeling scheme. Displacement ellipsoids are scaled to the 50\% probability level. 
Table S3. Crystal data and structure refinement for $\mathbf{1 c}$.

Chemical formula C40 H22 Br2 N2 O4

Formula weight 754.41

Temperature $123 \mathrm{~K}$

Wavelength $0.71073 \AA$

Crystal system Monoclinic

Space group $P 2{ }_{1} / n$

Unit cell dimensions $a=4.533(3) \AA$

$$
\begin{aligned}
& b=48.24(3) \AA \\
& c=13.863(8) \AA \\
& \beta=90.894(8)^{\circ}
\end{aligned}
$$

Volume 3031.0(3) $\AA^{3}$

$Z 4$

Density (calculated) $1.653 \mathrm{~g} \mathrm{~cm}^{-3}$

Absorption coefficient $2.725 \mathrm{~mm}^{-1}$

F (000) 1512

Crystal size $0.10 \times 0.03 \times 0.01 \mathrm{~mm}$

Theta range for data collection 1.69 to $22.98^{\circ}$

Index ranges $-4<=h<=4,-53<=k<=53,-15<=l<=15$

Reflections collected 24201

Independent reflections 4159

$R$ (int) $=0.3209$

Completeness to theta $=25.08^{\circ} 100 \%$

Absorption correction multi-scan

Max. and min. transmission 0.97 and 0.76

Refinement method SHELXL

Data / restraints / parameters 4159 / 108 / 482

Goodness-of-fit on F2 0.979

$\mathrm{R}$ indices $[\mathrm{I}>2 \operatorname{sigma}(\mathrm{I})] R 1=0.0676 w R 2=0.0786$

$\mathrm{R}$ indices (all data) $R 1=0.2119 w R 2=0.1076$

Largest diff. peak and hole 0.328 and -0.311 e $\AA^{-3}$

CCDC \#1991477 
Table S4. Cartesian coordinate of $\mathbf{1 a}$.

\begin{tabular}{|c|c|c|c|c|c|}
\hline \multirow{2}{*}{$\begin{array}{l}\text { Center } \\
\text { Number }\end{array}$} & \multirow{2}{*}{$\begin{array}{l}\text { Atomic } \\
\text { Number }\end{array}$} & \multirow{2}{*}{$\begin{array}{l}\text { Atomic } \\
\text { Type }\end{array}$} & \multicolumn{3}{|c|}{ Coordinates (Angstroms) } \\
\hline & & & $\mathrm{X}$ & $\mathrm{Y}$ & Z \\
\hline 1 & 8 & 0 & -3.392199 & -2.456470 & -1.198828 \\
\hline 2 & 8 & 0 & -5.855453 & 1.374526 & -1.027406 \\
\hline 3 & 7 & 0 & -4.877598 & -0.700466 & -1.320516 \\
\hline 4 & 6 & 0 & -2.744089 & -0.163486 & -0.618198 \\
\hline 5 & 6 & 0 & -1.358555 & -0.199495 & -0.298033 \\
\hline 6 & 6 & 0 & -3.487651 & 0.971513 & -0.546137 \\
\hline 7 & 6 & 0 & -1.556302 & 2.205490 & 0.264293 \\
\hline 8 & 6 & 0 & -0.725317 & 1.038498 & -0.031461 \\
\hline 9 & 6 & 0 & -0.646151 & -1.422498 & -0.202145 \\
\hline 10 & 6 & 0 & -3.637463 & -1.274473 & -1.071728 \\
\hline 11 & 6 & 0 & -7.953216 & -3.075550 & 1.832002 \\
\hline 12 & 6 & 0 & -4.882647 & 0.660525 & -0.973598 \\
\hline 13 & 6 & 0 & -6.757294 & -2.038488 & -0.471554 \\
\hline 14 & 6 & 0 & -8.208245 & -1.768539 & 1.440873 \\
\hline 15 & 6 & 0 & -1.066419 & 3.330471 & 0.962457 \\
\hline 16 & 6 & 0 & -2.955459 & 2.183499 & -0.034912 \\
\hline 17 & 6 & 0 & -7.613227 & -1.285509 & 0.291236 \\
\hline 18 & 6 & 0 & -6.504091 & -3.346500 & -0.056328 \\
\hline 19 & 6 & 0 & -6.072615 & -1.467456 & -1.681703 \\
\hline 20 & 6 & 0 & -3.765811 & 3.307492 & 0.241572 \\
\hline 21 & 6 & 0 & -7.101542 & -3.855530 & 1.075030 \\
\hline 22 & 6 & 0 & -1.881147 & 4.408464 & 1.229893 \\
\hline 23 & 6 & 0 & -3.215916 & 4.407475 & 0.838960 \\
\hline 24 & 8 & 0 & 3.392659 & -2.456537 & 1.198664 \\
\hline 25 & 8 & 0 & 5.855917 & 1.374467 & 1.027449 \\
\hline 26 & 7 & 0 & 4.877121 & -0.700541 & 1.320790 \\
\hline 27 & 6 & 0 & 2.744551 & -0.163521 & 0.618157 \\
\hline 28 & 6 & 0 & 1.359018 & -0.199512 & 0.297990 \\
\hline 29 & 6 & 0 & 3.488114 & 0.971481 & 0.546158 \\
\hline 30 & 6 & 0 & 1.556766 & 2.205503 & -0.264206 \\
\hline 31 & 6 & 0 & 0.724841 & 1.038496 & 0.031829 \\
\hline 32 & 6 & 0 & 0.646613 & -1.422509 & 0.202037 \\
\hline 33 & 6 & 0 & 3.637924 & -1.274533 & 1.071627 \\
\hline 34 & 6 & 0 & 7.952737 & -3.075456 & -1.831856 \\
\hline 35 & 6 & 0 & 4.881827 & 0.660469 & 0.973007 \\
\hline 36 & 6 & 0 & 6.756816 & -2.038518 & 0.471756 \\
\hline 37 & 6 & 0 & 8.207767 & -1.768467 & -1.440656 \\
\hline 38 & 6 & 0 & 1.065600 & 3.330522 & -0.962904 \\
\hline 39 & 6 & 0 & 2.955923 & 2.183495 & 0.034998 \\
\hline 40 & 6 & 0 & 7.612749 & -1.285498 & -0.290993 \\
\hline 41 & 6 & 0 & 6.504551 & -3.346507 & 0.056115 \\
\hline & & & & S23 & \\
\hline
\end{tabular}




\begin{tabular}{|c|c|c|c|c|c|}
\hline 42 & 6 & 0 & 6.073076 & -1.467551 & 1.681592 \\
\hline 43 & 6 & 0 & 3.765932 & 3.307502 & -0.242364 \\
\hline 44 & 6 & 0 & 7.101062 & -3.855477 & -1.074926 \\
\hline 45 & 6 & 0 & 1.880674 & 4.408529 & -1.229343 \\
\hline 46 & 6 & 0 & 3.216382 & 4.407518 & -0.838754 \\
\hline 47 & 1 & 0 & -8.384549 & -3.544573 & 2.704468 \\
\hline 48 & 1 & 0 & -1.074581 & -2.240491 & -0.421226 \\
\hline 49 & 1 & 0 & -8.783554 & -1.214553 & 1.955021 \\
\hline 50 & 1 & 0 & -0.162295 & 3.344463 & 1.253349 \\
\hline 51 & 1 & 0 & -7.804689 & -0.395501 & 0.018456 \\
\hline 52 & 1 & 0 & -5.911074 & -3.892486 & -0.559921 \\
\hline 53 & 1 & 0 & -5.815508 & -2.204439 & -2.291274 \\
\hline 54 & 1 & 0 & -6.702399 & -0.877443 & -2.165668 \\
\hline 55 & 1 & 0 & -4.687053 & 3.298498 & 0.014453 \\
\hline 56 & 1 & 0 & -6.924971 & -4.751537 & 1.336224 \\
\hline 57 & 1 & 0 & -1.526440 & 5.162452 & 1.686816 \\
\hline 58 & 1 & 0 & -3.750929 & 5.179471 & 0.989069 \\
\hline 59 & 1 & 0 & 8.384070 & -3.544433 & -2.704347 \\
\hline 60 & 1 & 0 & 1.073759 & -2.240515 & 0.420478 \\
\hline 61 & 1 & 0 & 8.783077 & -1.214453 & -1.954775 \\
\hline 62 & 1 & 0 & 0.162760 & 3.344531 & -1.253201 \\
\hline 63 & 1 & 0 & 7.804212 & -0.395505 & -0.018165 \\
\hline 64 & 1 & 0 & 5.911533 & -3.892520 & 0.559679 \\
\hline 65 & 1 & 0 & 5.814686 & -2.204567 & 2.290528 \\
\hline 66 & 1 & 0 & 6.702861 & -0.877564 & 2.165588 \\
\hline 67 & 1 & 0 & 4.686578 & 3.298496 & -0.013963 \\
\hline 68 & 1 & 0 & 6.925430 & -4.751469 & -1.336512 \\
\hline 69 & 1 & 0 & 1.525967 & 5.162542 & -1.686226 \\
\hline 70 & 1 & 0 & 3.750456 & 5.179522 & -0.988478 \\
\hline
\end{tabular}

Dipole moment (field-independent basis, Debye):

$\mathrm{X}=-0.0108 \quad \mathrm{Y}=2.9610 \quad \mathrm{Z}=-0.0020 \quad$ Tot $=2.9610$ 
Table S5. Cartesian coordinate of $\mathbf{1 b}$.

\begin{tabular}{|c|c|c|c|c|c|}
\hline \multirow{2}{*}{$\begin{array}{l}\text { Center } \\
\text { Number }\end{array}$} & \multirow{2}{*}{$\begin{array}{l}\text { Atomic } \\
\text { Number }\end{array}$} & \multirow{2}{*}{$\begin{array}{l}\text { Atomic } \\
\text { Type }\end{array}$} & \multicolumn{3}{|c|}{ Coordinates (Angstroms) } \\
\hline & & & $\mathrm{X}$ & $\mathrm{Y}$ & Z \\
\hline 1 & 8 & 0 & -3.248663 & -2.156113 & -1.547587 \\
\hline 2 & 8 & 0 & -8.760402 & -3.369204 & 2.030120 \\
\hline 3 & 8 & 0 & -5.716261 & 1.674922 & -1.635198 \\
\hline 4 & 7 & 0 & -4.713103 & -0.400082 & -1.824248 \\
\hline 5 & 6 & 0 & -2.663970 & 0.136851 & -0.902433 \\
\hline 6 & 6 & 0 & -1.319622 & 0.100814 & -0.438823 \\
\hline 7 & 6 & 0 & -3.410973 & 1.271861 & -0.908677 \\
\hline 8 & 6 & 0 & -1.575177 & 2.505793 & 0.099724 \\
\hline 9 & 6 & 0 & -0.717790 & 1.338791 & -0.107332 \\
\hline 10 & 6 & 0 & -0.621208 & -1.122203 & -0.268823 \\
\hline 11 & 1 & 0 & -1.024330 & -1.940186 & -0.531612 \\
\hline 12 & 6 & 0 & -3.505879 & -0.974114 & -1.446870 \\
\hline 13 & 6 & 0 & -8.101243 & -2.775166 & 0.988289 \\
\hline 14 & 6 & 0 & -4.754462 & 0.960903 & -1.479746 \\
\hline 15 & 6 & 0 & -6.670471 & -1.738087 & -1.177227 \\
\hline 16 & 6 & 0 & -8.313856 & -1.468145 & 0.572609 \\
\hline 17 & 1 & 0 & -8.939873 & -0.914157 & 1.023641 \\
\hline 18 & 6 & 0 & -1.161144 & 3.630754 & 0.845405 \\
\hline 19 & 1 & 0 & -0.292486 & 3.644726 & 1.229450 \\
\hline 20 & 6 & 0 & -2.935272 & 2.483830 & -0.344470 \\
\hline 21 & 6 & 0 & -7.601622 & -0.985105 & -0.508329 \\
\hline 22 & 1 & 0 & -7.763428 & -0.095090 & -0.799657 \\
\hline 23 & 6 & 0 & -6.462199 & -3.046109 & -0.737774 \\
\hline 24 & 1 & 0 & -5.819678 & -3.592097 & -1.176453 \\
\hline 25 & 6 & 0 & -5.863696 & -1.167046 & -2.308701 \\
\hline 26 & 1 & 0 & -5.544131 & -1.904024 & -2.887983 \\
\hline 27 & 1 & 0 & -6.439283 & -0.577015 & -2.855993 \\
\hline 28 & 6 & 0 & -3.770121 & 3.607831 & -0.154416 \\
\hline 29 & 1 & 0 & -4.662486 & 3.598857 & -0.476834 \\
\hline 30 & 6 & 0 & -7.174937 & -3.555148 & 0.324730 \\
\hline 31 & 1 & 0 & -7.026726 & -4.451162 & 0.602974 \\
\hline 32 & 6 & 0 & -9.721108 & -2.596224 & 2.733246 \\
\hline 33 & 1 & 0 & -9.285111 & -1.815247 & 3.131093 \\
\hline 34 & 1 & 0 & -10.122781 & -3.141250 & 3.441338 \\
\hline 35 & 1 & 0 & -10.419251 & -2.300187 & 2.112573 \\
\hline 36 & 6 & 0 & -1.999399 & 4.708757 & 1.026001 \\
\hline 37 & 1 & 0 & -1.694521 & 5.462731 & 1.517596 \\
\hline 38 & 6 & 0 & -3.285846 & 4.707796 & 0.497333 \\
\hline 39 & 1 & 0 & -3.833633 & 5.479799 & 0.590558 \\
\hline 40 & 8 & 0 & 3.248536 & -2.156332 & 1.547533 \\
\hline 41 & 8 & 0 & 8.759275 & -3.369240 & -2.030039 \\
\hline & & & & S25 & \\
\hline
\end{tabular}




\begin{tabular}{|c|c|c|c|c|c|}
\hline 42 & 8 & 0 & 5.716229 & 1.674634 & 1.635487 \\
\hline 43 & 7 & 0 & 4.712050 & -0.400362 & 1.824595 \\
\hline 44 & 6 & 0 & 2.664870 & 0.136704 & 0.902340 \\
\hline 45 & 6 & 0 & 1.320520 & 0.100742 & 0.438728 \\
\hline 46 & 6 & 0 & 3.411900 & 1.271695 & 0.908686 \\
\hline 47 & 6 & 0 & 1.576135 & 2.505763 & -0.099605 \\
\hline 48 & 6 & 0 & 0.717749 & 1.338764 & 0.107590 \\
\hline 49 & 6 & 0 & 0.622077 & -1.122242 & 0.268619 \\
\hline 50 & 1 & 0 & 1.023965 & -1.940259 & 0.530608 \\
\hline 51 & 6 & 0 & 3.505781 & -0.974330 & 1.446922 \\
\hline 52 & 6 & 0 & 8.100130 & -2.775279 & -0.988154 \\
\hline 53 & 6 & 0 & 4.753199 & 0.960653 & 1.479244 \\
\hline 54 & 6 & 0 & 6.670354 & -1.738358 & 1.177211 \\
\hline 55 & 6 & 0 & 8.313746 & -1.468300 & -0.572601 \\
\hline 56 & 1 & 0 & 8.938807 & -0.914287 & -1.023341 \\
\hline 57 & 6 & 0 & 1.160917 & 3.630801 & -0.845911 \\
\hline 58 & 1 & 0 & 0.292503 & 3.644829 & -1.228986 \\
\hline 59 & 6 & 0 & 2.936230 & 2.483726 & 0.344587 \\
\hline 60 & 6 & 0 & 7.601524 & -0.985339 & 0.508380 \\
\hline 61 & 1 & 0 & 7.763352 & -0.095354 & 0.799787 \\
\hline 62 & 6 & 0 & 6.463020 & -3.046335 & 0.737397 \\
\hline 63 & 1 & 0 & 5.820485 & -3.592347 & 1.176028 \\
\hline 64 & 6 & 0 & 5.863594 & -1.167398 & 2.308736 \\
\hline 65 & 1 & 0 & 5.542797 & -1.904420 & 2.887225 \\
\hline 66 & 1 & 0 & 6.439195 & -0.577430 & 2.856081 \\
\hline 67 & 6 & 0 & 3.770863 & 3.607724 & 0.153664 \\
\hline 68 & 1 & 0 & 4.662502 & 3.598699 & 0.477294 \\
\hline 69 & 6 & 0 & 7.174775 & -3.555297 & -0.324908 \\
\hline 70 & 1 & 0 & 7.027512 & -4.451282 & -0.603476 \\
\hline 71 & 6 & 0 & 9.720000 & -2.596221 & -2.733095 \\
\hline 72 & 1 & 0 & 9.284022 & -1.815198 & -3.130873 \\
\hline 73 & 1 & 0 & 10.122630 & -3.141194 & -3.441480 \\
\hline 74 & 1 & 0 & 10.418151 & -2.300257 & -2.112396 \\
\hline 75 & 6 & 0 & 1.999442 & 4.708799 & -1.025441 \\
\hline 76 & 1 & 0 & 1.693613 & 5.462825 & -1.516725 \\
\hline 77 & 6 & 0 & 3.286859 & 4.707759 & -0.497017 \\
\hline 78 & 1 & 0 & 3.833695 & 5.479757 & -0.589929 \\
\hline
\end{tabular}

Dipole moment (field-independent basis, Debye):

$\mathrm{X}=-0.0148 \quad \mathrm{Y}=4.6513 \quad \mathrm{Z}=-0.0029 \quad$ Tot $=4.6513$ 
Table S6. Cartesian coordinate of 1c.

\begin{tabular}{|c|c|c|c|c|c|}
\hline \multirow{2}{*}{$\begin{array}{l}\text { Center } \\
\text { Number }\end{array}$} & \multirow{2}{*}{$\begin{array}{l}\text { Atomic } \\
\text { Number }\end{array}$} & \multirow{2}{*}{$\begin{array}{l}\text { Atomic } \\
\text { Type }\end{array}$} & \multicolumn{3}{|c|}{ Coordinates (Angstroms) } \\
\hline & & & $\mathrm{X}$ & $\mathrm{Y}$ & Z \\
\hline 1 & 8 & 0 & 3.145538 & -1.753789 & 1.746332 \\
\hline 2 & 35 & 0 & 9.144696 & -3.192871 & -1.863313 \\
\hline 3 & 8 & 0 & 5.602955 & 2.077235 & 1.986959 \\
\hline 4 & 7 & 0 & 4.589992 & 0.002232 & 2.113364 \\
\hline 5 & 6 & 0 & 2.603001 & 0.539185 & 1.065980 \\
\hline 6 & 6 & 0 & 1.290015 & 0.503158 & 0.519834 \\
\hline 7 & 6 & 0 & 3.348180 & 1.674191 & 1.118585 \\
\hline 8 & 6 & 0 & 1.578508 & 2.908142 & -0.001787 \\
\hline 9 & 6 & 0 & 0.709920 & 1.741142 & 0.151647 \\
\hline 10 & 6 & 0 & 0.603494 & -0.719854 & 0.306804 \\
\hline 11 & 6 & 0 & 3.408513 & -0.571790 & 1.661786 \\
\hline 12 & 6 & 0 & 8.147122 & -2.372831 & -0.483695 \\
\hline 13 & 6 & 0 & 4.652655 & 1.363221 & 1.772109 \\
\hline 14 & 6 & 0 & 6.584719 & -1.335772 & 1.588865 \\
\hline 15 & 6 & 0 & 8.333533 & -1.065816 & -0.055606 \\
\hline 16 & 6 & 0 & 1.211554 & 4.033114 & -0.771711 \\
\hline 17 & 6 & 0 & 2.908415 & 2.886169 & 0.525958 \\
\hline 18 & 6 & 0 & 7.555590 & -0.582786 & 0.979052 \\
\hline 19 & 6 & 0 & 6.404117 & -2.643788 & 1.137319 \\
\hline 20 & 6 & 0 & 5.708300 & -0.764742 & 2.668280 \\
\hline 21 & 6 & 0 & 3.753452 & 4.010169 & 0.388094 \\
\hline 22 & 6 & 0 & 7.181419 & -3.152817 & 0.121089 \\
\hline 23 & 6 & 0 & 2.059403 & 5.111115 & -0.899925 \\
\hline 24 & 6 & 0 & 3.310562 & 5.110144 & -0.292439 \\
\hline 25 & 8 & 0 & -3.146071 & -1.753946 & -1.746220 \\
\hline 26 & 35 & 0 & -9.144227 & -3.192864 & 1.863339 \\
\hline 27 & 8 & 0 & -5.603537 & 2.077030 & -1.987102 \\
\hline 28 & 7 & 0 & -4.589564 & 0.002032 & -2.113552 \\
\hline 29 & 6 & 0 & -2.603563 & 0.539080 & -1.066021 \\
\hline 30 & 6 & 0 & -1.290577 & 0.503107 & -0.519872 \\
\hline 31 & 6 & 0 & -3.348756 & 1.674073 & -1.118701 \\
\hline 32 & 6 & 0 & -1.579100 & 2.908122 & 0.001588 \\
\hline 33 & 6 & 0 & -0.709514 & 1.741123 & -0.151950 \\
\hline 34 & 6 & 0 & -0.604040 & -0.719882 & -0.306760 \\
\hline 35 & 6 & 0 & -3.409061 & -0.571945 & -1.661753 \\
\hline 36 & 6 & 0 & -8.146663 & -2.372904 & 0.483666 \\
\hline 37 & 6 & 0 & -4.652061 & 1.363043 & -1.771404 \\
\hline 38 & 6 & 0 & -6.584274 & -1.335963 & -1.588963 \\
\hline 39 & 6 & 0 & -8.333091 & -1.065920 & 0.055490 \\
\hline 40 & 6 & 0 & -1.210995 & 4.033150 & 0.772238 \\
\hline 41 & 6 & 0 & -2.909007 & 2.886096 & -0.526155 \\
\hline & & & & S27 & \\
\hline
\end{tabular}




\begin{tabular}{|c|c|c|c|c|c|}
\hline 42 & 6 & 0 & -7.555154 & -0.582949 & -0.979201 \\
\hline 43 & 6 & 0 & -6.404638 & -2.643947 & -1.137146 \\
\hline 44 & 6 & 0 & -5.708845 & -0.764994 & -2.668233 \\
\hline 45 & 6 & 0 & -3.753876 & 4.010095 & -0.387384 \\
\hline 46 & 6 & 0 & -7.180950 & -3.152918 & -0.121066 \\
\hline 47 & 6 & 0 & -2.059041 & 5.111149 & 0.899396 \\
\hline 48 & 6 & 0 & -3.311183 & 5.110121 & 0.292093 \\
\hline 49 & 1 & 0 & 0.989528 & -1.537841 & 0.594094 \\
\hline 50 & 1 & 0 & 8.986336 & -0.511825 & -0.466912 \\
\hline 51 & 1 & 0 & 0.368404 & 4.047094 & -1.208925 \\
\hline 52 & 1 & 0 & 7.699007 & 0.307225 & 1.279871 \\
\hline 53 & 1 & 0 & 5.735608 & -3.189779 & 1.535271 \\
\hline 54 & 1 & 0 & 5.353398 & -1.501725 & 3.226604 \\
\hline 55 & 1 & 0 & 6.248813 & -0.174719 & 3.250245 \\
\hline 56 & 1 & 0 & 4.624088 & 4.001188 & 0.765271 \\
\hline 57 & 1 & 0 & 7.050758 & -4.048828 & -0.165828 \\
\hline 58 & 1 & 0 & 1.785624 & 5.865097 & -1.409484 \\
\hline 59 & 1 & 0 & 3.863081 & 5.882145 & -0.351480 \\
\hline 60 & 1 & 0 & -0.988897 & -1.537894 & -0.593196 \\
\hline 61 & 1 & 0 & -8.985901 & -0.511910 & 0.466759 \\
\hline 62 & 1 & 0 & -0.369011 & 4.047170 & 1.208650 \\
\hline 63 & 1 & 0 & -7.698583 & 0.307040 & -1.280079 \\
\hline 64 & 1 & 0 & -5.736122 & -3.189956 & -1.535062 \\
\hline 65 & 1 & 0 & -5.352768 & -1.502010 & -3.225708 \\
\hline 66 & 1 & 0 & -6.249366 & -0.175017 & -3.250238 \\
\hline 67 & 1 & 0 & -4.623712 & 4.001077 & -0.765725 \\
\hline 68 & 1 & 0 & -7.051261 & -4.048908 & 0.166094 \\
\hline 69 & 1 & 0 & -1.785271 & 5.865168 & 1.408904 \\
\hline 70 & 1 & 0 & -3.862728 & 5.882119 & 0.350899 \\
\hline
\end{tabular}

Dipole moment (field-independent basis, Debye):

$\mathrm{X}=0.0102 \quad \mathrm{Y}=4.7068 \quad \mathrm{Z}=0.0038 \quad$ Tot $=4.7068$ 


\section{References}

1. Wang, R.; Shi, K.; Cai, K.; Guo, Y.; Yang, X.; Wang, J.-Y.; Pei, J.; Zhao, D. Synthesis of polycyclic aromatic diimides via intramolecular cyclization of maleic acid derivatives. New J. Chem., 2016, 40, 113-121. DOI: 10.1039/c5nj01849h

2. Valera, J. S.; Gómez, R.; Sánchez L. Supramolecular Polymerization of [5]Helicenes. Consequences of Self-Assembly on Configurational Stability. Org. lett., 2018, 20, 2020-2023. DOI: 10.1021/acs.orglett.8b00565 